\title{
ZETA FUNCTIONS OF PERIODIC CUBICAL LATTICES AND CYCLOTOMIC-LIKE POLYNOMIALS
}

\author{
YASUAKI HIRAOKA, HIROYUKI OCHIAI, AND TOMOYUKI SHIRAI
}

\begin{abstract}
Zeta functions of periodic cubical lattices are explicitly derived by computing all the eigenvalues of the adjacency operators and their characteristic polynomials. We introduce cyclotomic-like polynomials to give factorization of the zeta function in terms of them and count the number of orbits of the Galois action associated with each cyclotomic-like polynomial to obtain its further factorization. We also give a necessary and sufficient condition for such a polynomial to be irreducible and discuss its irreducibility from this point of view.
\end{abstract}

\section{INTRODUCTION}

Let $K$ be an abstract simplicial complex over the set $K_{0}=\{1, \ldots, q\}$ of vertices, where we assume that the empty set $\emptyset$ is an element of $K$, i.e., $K_{-1}=\{\emptyset\}$. We denote the set of $k$-simplices and the $k$-dimensional skeleton of $K$ by $K_{k}$ and $K^{(k)}\left(=\sqcup_{i=-1}^{k} K_{i}\right)$. For each simplex $\sigma \in K,|\sigma|$ denotes the number of vertices in $\sigma$, and similarly, $|S|$ denotes the cardinality of a finite set $S$.

Let $\mathbf{n}=\left(n_{1}, \ldots, n_{q}\right) \in \mathbb{N}^{q}$ be a fixed vector of natural numbers with $n_{i} \geq 2$ for all $i \in K_{0}$. We write $|\mathbf{n}|=\prod_{i=1}^{q} n_{i}$. For each $i \in K_{0}$, let

$$
\mathcal{I}_{i}^{\circ}:=\left\{[0,1],[1,2], \ldots,\left[n_{i}-1,0\right]\right\}, \quad \mathcal{P}_{i}^{\circ}:=\left\{[0,0],[1,1], \ldots,\left[n_{i}-1, n_{i}-1\right]\right\}
$$

be the collections of intervals in $\mathbb{R} / n_{i} \mathbb{Z}$, where the elements of $\mathcal{P}_{i}^{\circ}$ are degenerated intervals. For each simplex $\sigma \in K$, we set a collection of $|\sigma|$-dimensional elementary cubes by

$$
Y_{\sigma}=\left\{I_{1} \times \cdots \times I_{q}: I_{i} \in \mathcal{I}_{i}^{\circ} \text { if } i \in \sigma ; I_{i} \in \mathcal{P}_{i}^{\circ} \text { otherwise }\right\} .
$$

Then, we define the $d$-cubical lattice $Y^{(d)}$ over $K$ by

$$
Y^{(d)}=\bigsqcup_{k=0}^{d} Y_{k}, \quad Y_{k}=\bigsqcup_{\sigma \in K_{k-1}} Y_{\sigma}
$$

for $d \leq q$. We also write $Y=Y^{(q)}$. When we need to specify the side lengths $\mathbf{n}$, we write $Y=Y(\mathbf{n})$. We refer to an element in $Y_{k}$ as a $k$-cube. The cubical lattice $Y$ is realized in the $q$-dimensional torus $\mathbb{T}^{q}$. For more explanation about elementary cubes (and also cubical homology), we refer the reader to [4]; in particular, see subsection 2.1.1. for elementary cubes.

A closed path in $Y^{(d)}$ is an alternating sequence $c=\left(\tau_{0}, \sigma_{0}, \tau_{1}, \sigma_{1}, \ldots, \tau_{n-1}, \sigma_{n-1}\right)$ of $(d-1)$-cubes $\left(\tau_{i}\right)_{i=0}^{n-1}$ and $d$-cubes $\left(\sigma_{i}\right)_{i=0}^{n-1}$ such that $\tau_{i} \neq \tau_{i+1}$ and $\tau_{i+1} \subset \sigma_{i} \cap \sigma_{i+1}$ for all $i \in \mathbb{Z}_{n}:=\mathbb{Z} / n \mathbb{Z}$. Note that $\tau_{0} \subset \sigma_{n-1} \cap \sigma_{0}$. The length of a closed path $c$, denoted by $|c|$, is the number of $d$-cubes (also $(d-1)$-cubes) in $c$. We say that a closed path $c$ is a closed geodesic if there is no back-tracking, i.e., $\sigma_{i} \neq \sigma_{i+1}$ for all $i \in \mathbb{Z}_{|c|}$. We denote by $c^{m}$ the $m$-multiple of a closed geodesic $c$, which is formed by $m$ repetitions of $c$. If

2010 Mathematics Subject Classification. 11R09, 11S40, 05E45, 05C50, 58C40.

Key words and phrases. Cubical lattice, zeta function, Laplacian, cyclotomic-like polynomial. 
a closed geodesic $c$ is not expressed as an $m$-multiple of a closed geodesic with $m \geq 2$, then $c$ is said to be prime. Two prime closed geodesics are said to be equivalent if one is obtained from the other through a cyclic permutation. An equivalence class of prime closed geodesics is called a prime cycle. The length of a prime cycle $\mathfrak{p}$ is the length of a representative and is denoted by $|\mathfrak{p}|$. The (Ihara) zeta function of a finite cubical lattice is defined as follows.

Definition 1.1. For $u \in \mathbb{C}$ with $|u|$ sufficient small, the (Ihara) zeta function of the $d$-skeleton $Y^{(d)}$ of a cubical lattice $Y$ is defined by

$$
\zeta_{Y^{(d)}}(u)=\prod_{\mathfrak{p} \in P_{d}}\left(1-u^{|\mathfrak{p}|}\right)^{-1}
$$

where $P_{d}$ is the set of prime cycles of $Y^{(d)}$. In particular, we write $\zeta_{Y}(u)$ for $\zeta_{Y^{(q)}}(u)$.

Although zeta functions can be defined in this manner for any cubical (simplicial) complexes, in the present paper, we only consider zeta functions for the cubical lattice $Y$ with the complete complex $K$, which includes all the subsets of $K_{0}$.

By symmetry, we have $\zeta_{Y^{(d)}}(u)=\zeta_{Y^{(q-d+1)}}(u)$ for $d=1,2, \ldots, q$ (see Remark 3.6)). For $d=q, q-1$ (and hence, by symmetry, for $d=1,2$, respectively), we have explicit factorizations of the zeta functions defined above.

Theorem 1.2. Let $Y$ be the periodic $q$-cubical lattice with side lengths $\mathbf{n}=\left(n_{1}, n_{2}, \ldots, n_{q}\right)$. Then, for $d=q$,

$$
\zeta_{Y}(u)^{-1}=\left(1-u^{2}\right)^{(q-1)|\mathbf{n}|} \prod_{k_{1}=1}^{n_{1}} \cdots \prod_{k_{q}=1}^{n_{q}}\left\{1-2 u \sum_{i=1}^{q} \cos \frac{2 \pi k_{i}}{n_{i}}+(2 q-1) u^{2}\right\}
$$

and for $d=q-1$,

$$
\zeta_{Y^{(q-1)}}(u)^{-1}=(1-u)^{\kappa|\mathbf{n}|}(1+3 u)^{\gamma|\mathbf{n}|} \times \prod_{k_{1}=1}^{n_{1}} \cdots \prod_{k_{q}=1}^{n_{q}} F_{1}^{\mathrm{up}}(u, \mathbf{k}) .
$$

Here $\mathbf{k}=\left(k_{1}, \ldots, k_{q}\right), \kappa=q(3 q-5) / 2, \gamma=q(q-3) / 2$ and

$$
F_{1}^{\mathrm{up}}(u, \mathbf{k})=\sum_{\ell=0}^{q}(2-\ell) 2^{\ell-1} e_{\ell}(\mathbf{w})\left\{1-2 u \sum_{i=1}^{q} \cos \frac{2 \pi k_{i}}{n_{i}}+3(2 q-3) u^{2}\right\}^{q-\ell} u^{\ell},
$$

where $e_{\ell}(\mathbf{t})$ is the $k$ th elementary symmetric polynomial in $\mathbf{t}=\left(t_{1}, \ldots, t_{q}\right)$, and $\mathbf{w}=$ $\left(2+2 \cos \frac{2 \pi k_{i}}{n_{i}}\right)_{i=1}^{q}$.

In principle, the expression of $\zeta_{Y^{(d)}}^{-1}$ for arbitrary $d$ can be obtained using Theorem 3.5 .

Let $Y=Y(\mathbf{n})$ be the periodic cubical lattice with side lengths $\mathbf{n}=\left(n_{1}, \ldots, n_{q}\right)$. Then, for $|u|<1$

$$
\begin{aligned}
& \lim _{n_{1}, \ldots, n_{q} \rightarrow \infty} \frac{-1}{|\mathbf{n}|} \log \zeta_{Y}(u) \\
& =(q-1) \log \left(1-u^{2}\right)+\int_{[0,1]^{q}} \log \left(1-2 u \sum_{i=1}^{q} \cos 2 \pi \theta_{i}+(2 q-1) u^{2}\right) d \theta_{1} \cdots d \theta_{q} .
\end{aligned}
$$

Note that the second term on the right-hand side is the logarithmic Mahler measure of the Laurant polynomial $1+(2 q-1) u^{2}-u \sum_{i=1}^{q}\left(z_{i}+z_{i}^{-1}\right) \in \mathbb{C}\left[z_{1}, z_{1}^{-1}, \ldots, z_{q}, z_{q}^{-1}\right]$ (cf. [2]). It is known that as $u \rightarrow 1$, the second term converges to 0 and $-4 G / \pi$ for $q=1$ and 2 , respectively, where $G$ is the Catalan number defined by $G=\sum_{k=0}^{\infty}(-1)^{k} /(2 k+1)^{2}$. 
The zeta function given in Theorem 1.2 can be written as a product of cyclotomic-like polynomials with integer coefficients. Let

$$
J_{1}=J_{2}=\{1\}, \quad J_{d}=\{j \in \mathbb{N}: j<d / 2, \operatorname{gcd}(j, d)=1\} \text { for } d \geq 3,
$$

and then

$$
\left|J_{d}\right|=\tilde{\varphi}(d):= \begin{cases}\varphi(d) / 2 & \text { for } d \geq 3, \\ 1 & \text { for } d=1,2,\end{cases}
$$

where $\varphi$ is the Euler function, i.e., $\varphi(d)$ is the cardinality of $\{j \in \mathbb{N}: j \leq d, \operatorname{gcd}(j, d)=1\}$. The set $J_{d}$ can be regarded as a set of representatives of $(\mathbb{Z} / d \mathbb{Z})^{\times} / \sim$, where $\sim$ is the equivalence relation with respect to the involution $\iota:(\mathbb{Z} / d \mathbb{Z})^{\times} \rightarrow(\mathbb{Z} / d \mathbb{Z})^{\times}$defined by $\iota(k)=d-k \bmod d$.

For $\mathbf{d}=\left(d_{1}, \ldots, d_{q}\right) \in \mathbb{N}^{q}$, we define the following homogeneous polynomial of $x$ and $y$ of degree $\prod_{i=1}^{q} \tilde{\varphi}\left(d_{i}\right)$ :

$$
\Psi_{\mathbf{d}}(x, y):=\prod_{j_{1} \in J_{d_{1}}} \cdots \prod_{j_{q} \in J_{d_{q}}}\left(x-2 y \sum_{i=1}^{q} \cos \frac{2 \pi j_{i}}{d_{i}}\right) .
$$

Then, it is seen that $\Psi_{\mathbf{d}}(x, y) \in \mathbb{Z}[x, y]$ (Proposition 4.4), and in particular, for $q=1$, $\Psi_{d}(x, y)$ is irreducible for any $d \in \mathbb{N}$ (Lemma 4.1) (cf. [7]). Although $\Psi_{\mathbf{d}}(x, y)$ may be reducible for general $\mathbf{d}$ unless $q=1$, if $\mathbf{d}=\left(d_{1}, \ldots, d_{q}\right)$ are relatively prime, then $\Psi_{\mathbf{d}}$ is irreducible (Corollary 4.5).

Using the polynomials $\Psi_{\mathbf{d}}$, we can factorize the zeta functions as follows.

Corollary 1.3. Let $Y$ be the periodic $q$-cubical lattice with side lengths $\mathbf{n}=\left(n_{1}, n_{2}, \ldots, n_{q}\right)$. Then,

$$
\zeta_{Y}(u)^{-1}=\left(1-u^{2}\right)^{(q-1)|\mathbf{n}|} \prod_{\mathbf{d} \mid \mathbf{n}} \Psi_{\mathbf{d}}\left(1+(2 q-1) u^{2}, u\right)^{\epsilon(\mathbf{d})}
$$

where $\mathbf{d} \mid \mathbf{n}$ means $d_{i} \mid n_{i}$ for all $i=1,2, \ldots, q$ and $\epsilon(\mathbf{d})=2^{k}$ with $k=\#\left\{1 \leq i \leq q: d_{i} \geq 3\right\}$.

For $\mathbf{d}=\left(d_{1}, \ldots, d_{q}\right)$, let $V=V_{\mathbf{d}}=\left\{j \in\{1,2, \ldots, q\}: d_{j} \geq 3\right\}$. We define a graph $\Gamma(V)$ obtained from $V$ by adding an unoriented edge between each pair of distinct $i$ and $j$ satisfying $\operatorname{gcd}\left(d_{i}, d_{j}\right) \geq 3$. We denote by $\tilde{\beta}_{0}(\Gamma(V))$ the 0 th reduced Betti number, i.e., one less than the number of connected components of $\Gamma(V)$. We understand $\tilde{\beta}_{0}(\Gamma(\emptyset))=0$ when $V=\emptyset$. Then we have the following.

Theorem 1.4. For $\mathbf{d}=\left(d_{1}, d_{2}, \ldots, d_{q}\right)$, let $N=d_{1} \cdots d_{q}$ and denote the number of $(\mathbb{Z} / N \mathbb{Z})^{\times}$-orbits in $J_{d_{1}} \times \cdots \times J_{d_{q}}$ by orb $(\mathbf{d})$, where the $(\mathbb{Z} / N \mathbb{Z})^{\times}$acts on $J_{d_{1}} \times \cdots \times J_{d_{q}}$ as the component-wise mulitiplication, i.e., $a \cdot\left(j_{1}, \ldots, j_{q}\right):=\left(a j_{1}, \ldots, a j_{q}\right)$ for $a \in(\mathbb{Z} / N \mathbb{Z})^{\times}$. Then,

$$
\operatorname{orb}(\mathbf{d})=\frac{\prod_{i=1}^{q} \tilde{\varphi}\left(d_{i}\right)}{\tilde{\varphi}\left(\operatorname{lcm}\left(d_{1}, d_{2}, \ldots, d_{q}\right)\right)} \times 2^{\tilde{\beta}_{0}\left(\Gamma\left(V_{\mathbf{d}}\right)\right)}
$$

While the above is the general form for the number of orbits, in certain special cases, this is greatly simplified as seen below.

Example 1.5. (i) For $q=2$, orb $\left(d_{1}, d_{2}\right)=\tilde{\varphi}\left(\operatorname{gcd}\left(d_{1}, d_{2}\right)\right)$.

(ii) For $d_{1}=d_{2}=\cdots=d_{q}=d, \operatorname{orb}(\mathbf{d})=\tilde{\varphi}(d)^{q-1}$. 
Next, for the purpose of factorizing $\Psi_{\mathbf{d}}(x)$, we introduce a new polynomial. For a subset $\mathcal{O} \subset J_{d_{1}} \times \cdots \times J_{d_{q}}$, we define

$$
\Psi_{\mathbf{d}}(x ; \mathcal{O}):=\prod_{\left(j_{1}, \ldots, j_{q}\right) \in \mathcal{O}}\left(x-2 \sum_{i=1}^{q} \cos \frac{2 \pi j_{i}}{d_{i}}\right) .
$$

It is obvious that this function has the following multiplicativity for the disjoint union:

$$
\Psi_{\mathbf{d}}\left(x ; \mathcal{O}_{1} \sqcup \mathcal{O}_{2}\right)=\Psi_{\mathbf{d}}\left(x ; \mathcal{O}_{1}\right) \Psi_{\mathbf{d}}\left(x ; \mathcal{O}_{2}\right) .
$$

From Theorem 1.4, we obtain the following factorization.

Corollary 1.6. Let $\sqcup_{i=1}^{\text {orb }(\mathbf{d})} \mathcal{O}_{i}$ be the decomposition of $J_{d_{1}} \times \cdots \times J_{d_{q}}$ into $(\mathbb{Z} / N \mathbb{Z})^{\times}$-orbits. Then,

$$
\Psi_{\mathbf{d}}(x)=\prod_{i=1}^{\operatorname{orb}(\mathbf{d})} \Psi_{\mathbf{d}}\left(x ; \mathcal{O}_{i}\right) .
$$

The degree of each $\Psi_{\mathbf{d}}\left(x ; \mathcal{O}_{i}\right) \in \mathbb{Z}[x]$ is $\tilde{\varphi}\left(\operatorname{lcm}\left(d_{1}, \ldots, d_{q}\right)\right) 2^{-\tilde{\beta}_{0}\left(\Gamma\left(V_{\mathbf{d}}\right)\right)}$.

The polynomials $\Psi_{\mathbf{d}}\left(x ; \mathcal{O}_{i}\right)$ may themselves be reducible. A complete characterization of the irreducibility of $\Psi_{\mathbf{d}}\left(x ; \mathcal{O}_{i}\right)$ remains open so far. Further discussion of this point is given in Section 4 .

This paper is organized as follows. In Section 2, we compute the eigenvalues of adjacency operators and Laplacians defined on the periodic cubical lattice. In Section 3, we recall the results for zeta functions of hypergraphs, and we present the results for cubical complexes in the cases $d=q, q-1$. In Section 4, we prove Theorem 1.4 and give a condition for $\Psi_{\mathbf{d}}(x ; \mathcal{O})$ to be irreducible (Proposition 4.4). Also, we give further discussion of factorization and present some observations for the case $q=2$.

\section{EigenVAlues FOR PERIODIC CUBICAL COMPLEXES}

2.1. Eigenvalues of adjacency matrices. The set $Y_{d}=\sqcup_{\sigma \in K_{d-1}} Y_{\sigma}$ of $d$-cubes (i.e., $|\sigma|=d)$ can be regarded as the set $K_{d-1} \times \mathbb{Z}_{n_{1}} \times \cdots \times \mathbb{Z}_{n_{q}}$ if we identify the intervals $I_{i}=\left[v_{i}, v_{i}+1\right] \in \mathcal{I}_{i}^{\circ}$ and $I_{i}=\left[v_{i}, v_{i}\right] \in \mathcal{P}_{i}^{\circ}$ with the points $v_{i} \in \mathbb{Z}_{n_{i}}$ as follows:

$$
\begin{aligned}
Y_{d} & =\bigsqcup_{\sigma \in K_{d-1}}\left\{I_{1} \times \cdots \times I_{q} \subset \mathbb{T}^{q}: I_{i} \in \mathcal{I}_{i}^{\circ} \text { if } i \in \sigma ; I_{i} \in \mathcal{P}_{i}^{\circ} \text { otherwise }\right\} \\
& \cong\left\{\left(\sigma, v_{1}, \ldots, v_{q}\right): \sigma \in K_{d-1}, v_{i} \in \mathbb{Z}_{n_{i}} \text { for } i \in K_{0}\right\}
\end{aligned}
$$

Here, note that $\left|Y_{d}\right|=\left(\begin{array}{c}q \\ d\end{array}\right)|\mathbf{n}|$.

We write $V:=\mathbb{Z}_{n_{1}} \times \cdots \times \mathbb{Z}_{n_{q}}$. For $d=0,1, \ldots, q$, the space $C^{d}(Y)$ of $d$-cochains on $Y$ is regarded as $C^{d}\left(K_{d-1} \times V\right)$, the space of functions on $K_{d-1} \times V$, under the identification introduced above. We continue to make this identification below although we use the notation $C^{d}(Y)$.

Now, we define the incidence operator $M_{d}: C^{d}(Y) \rightarrow C^{d+1}(Y)$ by

$$
M_{d} f(\eta, \mathbf{v})=\sum_{j \in \eta}\left\{f\left(\eta_{j}, \mathbf{v}\right)+f\left(\eta_{j}, S_{j} \mathbf{v}\right)\right\} \quad \text { for } \eta \in K_{d},
$$

where $\eta_{j}=\eta \backslash\{j\}, \mathbf{v}=\left(v_{1}, \ldots, v_{q}\right) \in V$ and $S_{j} \mathbf{v}=\left(v_{1}, \ldots, v_{j}+1, \ldots, v_{q}\right)$. Equivalently, we can write $M_{d}$ as

$$
M_{d} f(\eta, \mathbf{v})=\sum_{\sigma \in K_{d-1}: \sigma \subset \eta}\left\{f(\sigma, \mathbf{v})+f\left(\sigma, S_{\eta \backslash \sigma} \mathbf{v}\right)\right\} \quad \text { for } \eta \in K_{d} .
$$


The matrix representation of $M_{d}$ is nothing but the incidence matrix between $Y_{d}$ and $Y_{d-1}$. The dual operator $M_{d}^{*}: C^{d+1}(Y) \rightarrow C^{d}(Y)$ of $M_{d}$ with respect to the inner product

$$
\langle f, g\rangle_{C^{d}(Y)}:=|V|^{-1} \sum_{\sigma \in K_{d-1}} \sum_{\mathbf{v} \in V} f(\sigma, \mathbf{v}) \overline{g(\sigma, \mathbf{v})}
$$

is given by

$$
M_{d}^{*} f(\sigma, \mathbf{v})=\sum_{\eta \in K_{d}: \eta \supset \sigma}\left\{f(\eta, \mathbf{v})+f\left(\eta, S_{\eta \backslash \sigma}^{-1} \mathbf{v}\right)\right\} \quad \text { for } \sigma \in K_{d-1}
$$

Let $\hat{\mathbb{Z}}_{n_{j}}$ be the character group of $\mathbb{Z}_{n_{j}}$. An element $z_{j}$ of $\hat{\mathbb{Z}}_{n_{j}}$ is expressed as $z_{j}=$ $\exp \left(2 \pi \sqrt{-1} k_{j} / n_{j}\right)$ for some $k_{j} \in \mathbb{Z}_{n_{j}}$. We write $\hat{V}:=\hat{\mathbb{Z}}_{n_{1}} \times \cdots \times \hat{\mathbb{Z}}_{n_{q}}$. For $\mathbf{z}=\left(z_{1}, \ldots, z_{q}\right) \in$ $\hat{V}$, we consider a subspace $C_{\mathbf{z}}^{d}$ of $C^{d}(Y)$ defined by

$$
C_{\mathbf{z}}^{d}:=C_{\mathbf{z}}^{d}(Y):=\left\{f \in C^{d}(Y): S_{j} f=z_{j} f \text { for any } j \in K_{0}\right\},
$$

where $S_{j} f(\sigma, \mathbf{v}):=f\left(\sigma, S_{j} \mathbf{v}\right)$. It is clear that $\operatorname{dim} C_{\mathbf{z}}^{d}=\left|K_{d-1}\right|=\left(\begin{array}{l}q \\ d\end{array}\right)$, and $C_{\mathbf{z}}^{d}$ and $C_{\mathbf{w}}^{d}$ are orthogonal unless $\mathbf{z}=\mathbf{w}$. Hence, we have the orthogonal decomposition

$$
C^{d}(Y)=\bigoplus_{\mathbf{z} \in \hat{V}} C_{\mathbf{z}}^{d}
$$

For $\mathbf{z}=\left(z_{1}, \ldots, z_{q}\right) \in \hat{V}$, we also consider the map $U_{\mathbf{z}}: C^{d}\left(K_{d-1}\right) \rightarrow C_{\mathbf{z}}^{d}(Y)$ defined by

$$
U_{\mathbf{z}} f(\sigma, \mathbf{v})=f(\sigma) \mathbf{z}^{\mathbf{v}} \quad\left(\sigma \in K_{d-1}, \mathbf{v} \in V\right),
$$

where $\mathbf{z}^{\mathbf{v}}=\prod_{j=1}^{q} z_{j}^{v_{j}}$. Then, it is easy to see that $\left\langle U_{\mathbf{z}} f, U_{\mathbf{z}} g\right\rangle_{C^{d}(Y)}=\langle f, g\rangle_{C^{d}\left(K_{d-1}\right)}$, i.e., $U_{\mathbf{z}}$ is unitary. The inverse map $U_{\mathbf{z}}^{-1}: C_{\mathbf{z}}^{d}(Y) \rightarrow C^{d}\left(K_{d-1}\right)$ is the finite Fourier transform given by

$$
\left(U_{\mathbf{z}}^{-1} f\right)(\sigma)=\frac{1}{|V|} \sum_{\mathbf{v} \in V} f(\sigma, \mathbf{v}) \mathbf{z}^{-\mathbf{v}} \quad \text { for } \sigma \in K_{d-1} .
$$

Since $f(\sigma, \mathbf{v}) \in C_{\mathbf{z}}^{d}$, we see that $\left(U_{\mathbf{z}}^{-1} f\right)(\sigma)=f(\sigma, \mathbf{0})$.

Lemma 2.1. For $d=0,1, \ldots, q-1$, the operator $M_{d}$ (also $M_{d}^{*}$ ) preserves each fiber of z, i.e., $M_{d} C_{\mathbf{z}}^{d} \subset C_{\mathbf{z}}^{d+1}$ (also $M_{d}^{*} C_{\mathbf{z}}^{d+1} \subset C_{\mathbf{z}}^{d}$ ).

Proof. It is easily verified.

The restriction $M_{d}$ on each fiber $\mathbf{z}$ is called the twisted operator of $M_{d}$ and denoted by $M_{d}(\mathbf{z})$. Thus, we have the following direct sum decomposition:

$$
M_{d}=\bigoplus_{\mathbf{z} \in \hat{V}} M_{d}(\mathbf{z}): \bigoplus_{\mathbf{z} \in \hat{V}} C_{\mathbf{z}}^{d} \rightarrow \bigoplus_{\mathbf{z} \in \hat{V}} C_{\mathbf{z}}^{d+1} .
$$

The situation for $M_{d}^{*}: C^{d+1}(Y) \rightarrow C^{d}(Y)$ is similar.

Lemma 2.2. For $\mathrm{z} \in \hat{V}$,

$$
\begin{aligned}
& \left(M_{d}(\mathbf{z}) f\right)(\eta, \mathbf{v})=\sum_{\sigma \in K_{d-1}: \sigma \subset \eta}\left(1+z_{\eta \backslash \sigma}\right) f(\sigma, \mathbf{v}), \\
& \left(M_{d}^{*}(\mathbf{z}) f\right)(\sigma, \mathbf{v})=\sum_{\substack{\eta \in K_{d}: \eta \supset \sigma \\
5}}\left(1+z_{\eta \backslash \sigma}^{-1}\right) f(\eta, \mathbf{v}) .
\end{aligned}
$$


Proof. By the definition of $M_{d}$ and $f \in C_{\mathbf{z}}^{d}$, we see that

$$
\begin{aligned}
M_{d}(\mathbf{z}) f(\eta, \mathbf{v}) & =\sum_{\sigma \in K_{d-1}: \sigma \subset \eta}\left\{f(\sigma, \mathbf{v})+f\left(\sigma, S_{\eta \backslash \sigma} \mathbf{v}\right)\right\} \\
& =\sum_{\sigma \in K_{d-1}: \sigma \subset \eta}\left(1+z_{\eta \backslash \sigma}\right) f(\sigma, \mathbf{v}) .
\end{aligned}
$$

The proof for $M_{d}^{*}(\mathbf{z})$ is similar.

The operator $\tilde{M}_{d}(\mathbf{z}):=U_{\mathbf{z}}^{-1} M_{d}(\mathbf{z}) U_{\mathbf{z}}: C^{d}\left(K_{d-1}\right) \rightarrow C^{d+1}\left(K_{d}\right)$ is unitarily equivalent to $M_{d}(\mathbf{z})$ so that we abuse the notation $M_{d}(\mathbf{z})$ for $\tilde{M}_{d}(\mathbf{z})$ in what follows.

The matrix representation of $M_{d}(\mathbf{z})$ is the $\left|K_{d}\right| \times\left|K_{d-1}\right|$-matrix whose $(\eta, \sigma)$-element is given by $\left(1+z_{\eta \backslash \sigma}\right) \mathbf{1}(\sigma \subset \eta)$, where $\mathbf{1}(\sigma \subset \eta)=1$ if $\sigma \subset \eta$; 0 otherwise. Similarly, the matrix representation of $M_{d}^{*}(\mathbf{z})$ is the $\left|K_{d-1}\right| \times\left|K_{d}\right|$-matrix whose $(\sigma, \eta)$-element is given by $\left(1+z_{\eta \backslash \sigma}^{-1}\right) \mathbf{1}(\sigma \subset \eta)$.

Let $A_{d}^{\text {up }}=M_{d}^{*} M_{d}$ and $A_{d}^{\text {down }}=M_{d-1} M_{d-1}^{*}$, both acting on $C^{d}(Y)$. They also have the direct sum decompositions

$$
A_{d}^{\text {up }}=\oplus_{\mathbf{z} \in \hat{V}} A_{d}^{\text {up }}(\mathbf{z}), A_{d}^{\text {down }}=\oplus_{\mathbf{z} \in \hat{V}} A_{d}^{\text {down }}(\mathbf{z}),
$$

where $A_{d}^{\text {up }}(\mathbf{z})=M_{d}^{*}(\mathbf{z}) M_{d}(\mathbf{z})$ and $A_{d}^{\text {down }}(\mathbf{z})=M_{d-1}(\mathbf{z}) M_{d-1}^{*}(\mathbf{z})$ act on $C_{\mathbf{z}}^{d}$.

In the following lemma, we use the metric on $K_{d-1}$ defined by $\rho\left(\sigma, \sigma^{\prime}\right):=\left|\sigma \backslash \sigma^{\prime}\right|(=$ $\left.\left|\sigma^{\prime} \backslash \sigma\right|\right)$.

Lemma 2.3. The $\left(\sigma, \sigma^{\prime}\right)$-element of the matrix representation of $A_{d}^{\mathrm{up}}(\mathbf{z})$ is given by

$$
a_{\sigma \sigma^{\prime}}^{\text {up }}(\mathbf{z})= \begin{cases}\sum_{\eta \in K_{d}: \eta \supset \sigma}\left(1+z_{\eta \backslash \sigma}^{-1}\right)\left(1+z_{\eta \backslash \sigma}\right) & \text { if } \rho\left(\sigma, \sigma^{\prime}\right)=0\left(\text { i.e., } \sigma=\sigma^{\prime}\right), \\ \left(1+z_{\sigma^{\prime} \backslash \sigma}^{-1}\right)\left(1+z_{\sigma \backslash \sigma^{\prime}}\right) & \text { if } \rho\left(\sigma, \sigma^{\prime}\right)=1, \\ 0 & \text { if } \rho\left(\sigma, \sigma^{\prime}\right) \geq 2,\end{cases}
$$

and the set of eigenvalues of $A_{d}^{\text {up }}$ coincides with the union of the sets of eigenvalues of $A_{d}^{\mathrm{up}}(\mathbf{z})=\left(a_{\sigma \sigma^{\prime}}^{\mathrm{up}}(\mathbf{z})\right)_{\sigma, \sigma^{\prime} \in K_{d-1}}$ for $\mathbf{z} \in \hat{V}$, i.e.

$$
\operatorname{Spec}\left(A_{d}^{\text {up }}\right)=\bigsqcup_{\mathbf{z} \in \hat{V}} \operatorname{Spec}\left(A_{d}^{\text {up }}(\mathbf{z})\right) .
$$

Similarly, the $\left(\sigma, \sigma^{\prime}\right)$-element of the matrix representation of $A_{d}^{\text {down }}(\mathbf{z})$ is given by

$$
a_{\sigma \sigma^{\prime}}^{\text {down }}(\mathbf{z})= \begin{cases}\sum_{\tau \in K_{d-2}: \tau \subset \sigma}\left(1+z_{\sigma \backslash \tau}\right)\left(1+z_{\sigma \backslash \tau}^{-1}\right) & \text { if } \rho\left(\sigma, \sigma^{\prime}\right)=0, \\ \left(1+z_{\sigma \backslash \sigma^{\prime}}\right)\left(1+z_{\sigma^{\prime} \backslash \sigma}^{-1}\right) & \text { if } \rho\left(\sigma, \sigma^{\prime}\right)=1, \\ 0 & \text { if } \rho\left(\sigma, \sigma^{\prime}\right) \geq 2,\end{cases}
$$

and the set of eigenvalues of $A_{d}^{\text {down }}$ coincides with the union of the sets of eigenvalues of $A_{d}^{\text {down }}(\mathbf{z})=\left(a_{\sigma \sigma^{\prime}}^{\text {down }}(\mathbf{z})\right)_{\sigma, \sigma^{\prime} \in K_{d-1}}$ for $\mathbf{z} \in \hat{V}$, i.e.,

$$
\operatorname{Spec}\left(A_{d}^{\text {down }}\right)=\bigsqcup_{\substack{\mathbf{z} \in \hat{V} \\ 6}} \operatorname{Spec}\left(A_{d}^{\text {down }}(\mathbf{z})\right)
$$


Proof. For $\mathbf{z} \in \hat{V}$, we have

$$
\begin{aligned}
a_{\sigma \sigma^{\prime}}^{\text {up }}(\mathbf{z}) & =\sum_{\eta \in K_{d}}\left(1+z_{\eta \backslash \sigma}^{-1}\right)\left(1+z_{\eta \backslash \sigma^{\prime}}\right) \mathbf{1}\left(\eta \supset \sigma \cup \sigma^{\prime}\right), \\
a_{\sigma \sigma^{\prime}}^{\text {down }}(\mathbf{z}) & =\sum_{\tau \in K_{d-2}}\left(1+z_{\sigma \backslash \tau}\right)\left(1+z_{\sigma^{\prime} \backslash \tau}^{-1}\right) \mathbf{1}\left(\tau \subset \sigma \cap \sigma^{\prime}\right) .
\end{aligned}
$$

If $\rho\left(\sigma, \sigma^{\prime}\right) \geq 2$, then $\mathbf{1}\left(\eta \supset \sigma \cup \sigma^{\prime}\right)=\mathbf{1}\left(\tau \subset \sigma \cap \sigma^{\prime}\right)=0$. If $\rho\left(\sigma, \sigma^{\prime}\right)=1$, then $\eta$ (resp. $\tau$ ) must coincide with $\sigma \cup \sigma^{\prime}$ (resp. $\sigma \cap \sigma^{\prime}$ ) when $\eta \supset \sigma \cup \sigma^{\prime}$ (resp. $\tau \subset \sigma \cap \sigma^{\prime}$ ). We thus obtain the desired expressions.

Note that when $q \geq 2 d-1$, which is equivalent to $\left(\begin{array}{l}q \\ d\end{array}\right) \geq\left(\begin{array}{c}q \\ d-1\end{array}\right)$, we have

$$
\operatorname{Spec}\left(A_{d}^{\text {down }}(\mathbf{z})\right)=\operatorname{Spec}\left(A_{d-1}^{\text {up }}(\mathbf{z})\right) \cup\{0\},
$$

with the multiplicity of 0 being $\left(\begin{array}{l}q \\ d\end{array}\right)-\left(\begin{array}{c}q \\ d-1\end{array}\right)$, and similarly, when $q \leq 2 d-1$, we have

$$
\operatorname{Spec}\left(A_{d-1}^{\text {up }}(\mathbf{z})\right)=\operatorname{Spec}\left(A_{d}^{\text {down }}(\mathbf{z})\right) \cup\{0\},
$$

with the multiplicity of 0 being $\left(\begin{array}{c}q \\ d-1\end{array}\right)-\left(\begin{array}{l}q \\ d\end{array}\right)$.

Corollary 2.4. The eigenvalues of $A_{q}^{\text {down }}: C^{q}(Y) \rightarrow C^{q}(Y)$ on the periodic $q$-cubical lattice $Y$ are $\left\{\sum_{j=1}^{q} 2\left\{1+\cos \left(2 \pi k_{j} / n_{j}\right)\right\}: k_{j} \in \mathbb{Z}_{n_{j}}\right\}$.

Proof. From Lemma 2.3 , we know that $A_{q}^{\text {down }}(\mathbf{z})$ is a scalar and equal to $\sum_{j=1}^{q}\left(1+z_{j}^{-1}\right)(1+$ $\left.z_{j}\right)$. Therefore, the eigenvalues of $A_{q}^{\text {down }}$ are given by $\left\{\sum_{j=1}^{q}\left(1+z_{j}^{-1}\right)\left(1+z_{j}\right): z_{j} \in \hat{\mathbb{Z}}_{n_{j}}\right\}$. The assertion follows directly.

2.2. Eigenvalues of Laplacians. Techniques similar to those used in the previous section can be applied to eigenvalue problems of Laplacians.

For $\sigma, \tau \in K$ with $\tau \subset \sigma$ and $|\sigma \backslash \tau|=1$, we write $\operatorname{sgn}(\sigma, \tau)=(-1)^{j-1}$ if $\sigma \backslash \tau$ is in the $j$ th position of $\sigma$ in lexicographic order. For example, $\operatorname{sgn}(134,34)=1, \operatorname{sgn}(134,14)=-1$ and $\operatorname{sgn}(134,13)=1$.

For $d=0,1, \ldots, q-1$, let $\delta_{d}: C^{d}(Y) \rightarrow C^{d+1}(Y)$ be defined by

$$
\delta_{d} f(\eta, \mathbf{v})=\sum_{\sigma \in K_{d-1}: \sigma \subset \eta} \operatorname{sgn}(\eta, \sigma)\left\{f\left(\sigma, S_{\eta \backslash \sigma} \mathbf{v}\right)-f(\sigma, \mathbf{v})\right\} \quad \text { for } \eta \in K_{d} .
$$

The dual operator of $\delta_{d}, \delta_{d}^{*}$, is defined analogously to $M_{d}^{*}$ with respect to the inner product (2.1). As in the previous subsection, because both $\delta_{d}$ and $\delta_{d}^{*}$ preserve the fiber of $\mathbf{z}$, the operators $\delta_{d}$ and $\delta_{d}^{*}$ can be decomposed into the direct sums $\bigoplus_{\mathbf{z} \in \hat{V}} \delta_{d}(\mathbf{z})$ and $\bigoplus_{\mathbf{z} \in \hat{V}} \delta_{d}^{*}(\mathbf{z})$, respectively. As in the proof of Lemma 2.2 , we easily see that

$$
\begin{aligned}
& \delta_{d}(\mathbf{z}) f(\eta, \mathbf{v})=\sum_{\sigma \in K_{d-1}: \sigma \subset \eta} \operatorname{sgn}(\eta, \sigma)\left(z_{\eta \backslash \sigma}-1\right) f(\sigma, \mathbf{v}) \quad \text { for } \eta \in K_{d}, \\
& \delta_{d}^{*}(\mathbf{z}) f(\sigma, \mathbf{v})=\sum_{\eta \in K_{d}: \eta \supset \sigma} \operatorname{sgn}(\eta, \sigma)\left(z_{\eta \backslash \sigma}^{-1}-1\right) f(\eta, \mathbf{v}) \quad \text { for } \sigma \in K_{d-1} .
\end{aligned}
$$

Lemma 2.5. For $d=0,1, \ldots, q-1$, let $g: K_{0} \rightarrow \mathbb{C}$ and define $G_{d}: C^{d}(Y) \rightarrow C^{d+1}(Y)$ by

$$
G_{d} f(\eta)=\sum_{\sigma \in K_{d-1}: \sigma \subset \eta} \operatorname{sgn}(\eta, \sigma) g(\eta \backslash \sigma) f(\sigma) \quad\left(\eta \in K_{d}\right)
$$

Then, $G_{d+1} G_{d}=0$. In particular, $\delta_{d+1}(\mathbf{z}) \delta_{d}(\mathbf{z})=0$ for any $\mathbf{z} \in \hat{V}$. 
Proof. For $f \in C^{d}(Y)$,

$$
\begin{aligned}
\left(G_{d+1} G_{d} f\right)(\tau) & =\sum_{\sigma \in K_{d}: \sigma \subset \eta} \sum_{\tau \in K_{d-1}: \tau \subset \sigma} \operatorname{sgn}(\eta, \sigma) \operatorname{sgn}(\sigma, \tau) g(\eta \backslash \sigma) g(\sigma \backslash \tau) f(\tau) \\
& =\left(\sum_{1 \leq i<j \leq q} g(i) g(j) \sum_{\{\eta \backslash \sigma, \sigma \backslash \tau\}=\{i, j\}} \operatorname{sgn}(\eta, \sigma) \operatorname{sgn}(\sigma, \tau)\right) f(\tau) \\
& =0 .
\end{aligned}
$$

The last sum is taken separately for two cases, $(\eta \backslash \sigma, \sigma \backslash \tau)=(i, j)$ and $(j, i)$, which is equal to 0 as usual.

From this lemma, we have the cochain complex

$$
\cdots \rightarrow C_{\mathbf{z}}^{d-1} \stackrel{\delta_{d-1}(\mathbf{z})}{\rightarrow} C_{\mathbf{z}}^{d} \stackrel{\delta_{d}(\mathbf{z})}{\rightarrow} C_{\mathbf{z}}^{d+1} \rightarrow \cdots
$$

and hence the cohomology group $H_{\mathbf{z}}^{d}:=\operatorname{ker} \delta_{d}(\mathbf{z}) / \operatorname{Im} \delta_{d-1}(\mathbf{z})$ is defined.

Lemma 2.6. For all $d=0,1, \ldots, q-1$ and $\mathbf{z} \in \hat{V}$, the cohomology group $H_{\mathbf{z}}^{d}$ is trivial

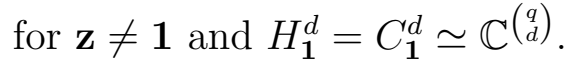

Proof. First, we note that $H^{d}(Y)=\oplus_{\mathbf{z} \in \hat{V}} H_{\mathbf{z}}^{d}$ is the $d$ th cohomology of the $q$-dimensional torus and is given by $H^{d}(Y)=\mathbb{C}^{\left(\begin{array}{l}q \\ d\end{array}\right)}$. For $\mathbf{z}=\mathbf{1}$, since $\delta_{d}(\mathbf{z})=0$, we have $H_{1}^{d}=C_{\mathbf{1}}^{d} \simeq \mathbb{C}^{\left(\begin{array}{l}q \\ d\end{array}\right)}$. Hence, $H_{\mathbf{z}}^{d}=0$ for $\mathbf{z} \neq \mathbf{1}$.

Corollary 2.7. If $\mathbf{z} \neq \mathbf{1}$, then $\operatorname{dim} \operatorname{ker} \delta_{d}(\mathbf{z})=\left(\begin{array}{c}q-1 \\ d-1\end{array}\right)$ and $\operatorname{dim} \operatorname{ker} \delta_{d-1}^{*}(\mathbf{z})=\left(\begin{array}{c}q-1 \\ d\end{array}\right)$ for $d=0,1, \ldots, q$. Here, $\left(\begin{array}{c}q-1 \\ -1\end{array}\right)=\left(\begin{array}{c}q-1 \\ q\end{array}\right)=0$.

Proof. From the rank-nullity theorem together with Lemma 2.6, we have

$$
\left(\begin{array}{l}
q \\
d
\end{array}\right)=\operatorname{dim} \operatorname{ker} \delta_{d}(\mathbf{z})+\operatorname{dim} \operatorname{ker} \delta_{d+1}(\mathbf{z}) \quad(d=0,1, \ldots, q-1) .
$$

Since $\operatorname{dim} \operatorname{ker} \delta_{0}(\mathbf{z})=0$, taking the alternating sum above yields $\operatorname{dim} \operatorname{ker} \delta_{d}(\mathbf{z})=\left(\begin{array}{c}q-1 \\ d-1\end{array}\right)$. Since $\operatorname{ker} \delta_{d-1}^{*}(\mathbf{z})=\left(\operatorname{Im} \delta_{d-1}(\mathbf{z})\right)^{\perp}=\left(\operatorname{ker} \delta_{d}(\mathbf{z})\right)^{\perp}$, from Lemma 2.6, we have

$$
C_{\mathbf{z}}^{d}=\operatorname{ker} \delta_{d}(\mathbf{z}) \oplus \operatorname{ker} \delta_{d-1}^{*}(\mathbf{z}) .
$$

Therefore, $\operatorname{dim} \operatorname{ker} \delta_{d-1}^{*}(\mathbf{z})=\left(\begin{array}{c}q-1 \\ d\end{array}\right)$.

Next, we give the matrix representations for the up/down Laplacians. Let $L_{d}^{\mathrm{up}}=\delta_{d}^{*} \delta_{d}$ and $L_{d}^{\text {down }}=\delta_{d-1} \delta_{d-1}^{*}$. These operators can be decomposed as $L_{d}^{\text {up }}=\oplus_{\mathbf{z} \in \hat{V}} L_{d}^{\text {up }}(\mathbf{z})$ and $L_{d}^{\text {down }}=\oplus_{\mathbf{z} \in \hat{V}} L_{d}^{\text {down }}(\mathbf{z})$. As in the case of Lemma 2.3, we have the following.

Lemma 2.8. The $\left(\sigma, \sigma^{\prime}\right)$-element of the matrix representation of $L_{d}^{\mathrm{up}}(\mathbf{z})$ is given by

$$
\ell_{\sigma \sigma^{\prime}}^{\text {up }}(\mathbf{z})=\sum_{\eta \in K_{d}: \eta \supset \sigma \cup \sigma^{\prime}} \operatorname{sgn}(\eta, \sigma) \operatorname{sgn}\left(\eta, \sigma^{\prime}\right)\left(z_{\eta \backslash \sigma}^{-1}-1\right)\left(z_{\eta \backslash \sigma^{\prime}}-1\right)
$$

and the $\left(\sigma, \sigma^{\prime}\right)$-element of the matrix representation of $L_{d}^{\text {down }}(\mathbf{z})$ is given by

$$
\ell_{\sigma \sigma^{\prime}}^{\text {down }}(\mathbf{z})=\sum_{\tau \in K_{d-2}: \tau \subset \sigma \cap \sigma^{\prime}} \operatorname{sgn}(\sigma, \tau) \operatorname{sgn}\left(\sigma^{\prime}, \tau\right)\left(z_{\sigma \backslash \tau}-1\right)\left(z_{\sigma^{\prime} \backslash \tau}^{-1}-1\right) .
$$

Corollary 2.9. Suppose $K$ is the complete simplicial complex over $\{1,2, \ldots, q\}$. Then, we have

$$
L_{d}^{\text {up }}(\mathbf{z})+L_{d}^{\text {down }}(\mathbf{z})=\left\{2 q-\sum_{i=1}^{q}\left(z_{i}+z_{i}^{-1}\right)\right\} I .
$$


Proof. In Lemma 2.8, we chose $\sigma, \sigma^{\prime} \in K_{d-1}$. If $\sigma \neq \sigma^{\prime}$, then $\ell_{\sigma \sigma^{\prime}}^{\text {up }}(\mathbf{z})=\ell_{\sigma \sigma^{\prime}}^{\text {down }}(\mathbf{z})=0$ unless $\rho\left(\sigma, \sigma^{\prime}\right)=1$. When $\rho\left(\sigma, \sigma^{\prime}\right)=1$, we see that $\tau=\sigma \cap \sigma^{\prime}, \eta=\sigma \cup \sigma^{\prime}, \sigma \backslash \tau=\eta \backslash \sigma^{\prime}$ and $\sigma^{\prime} \backslash \tau=\eta \backslash \sigma$. Using the same argument as in the proof of Lemma 2.5, we obtain

$$
\operatorname{sgn}\left(\sigma \cup \sigma^{\prime}, \sigma\right) \operatorname{sgn}\left(\sigma \cup \sigma^{\prime}, \sigma^{\prime}\right)+\operatorname{sgn}\left(\sigma, \sigma \cap \sigma^{\prime}\right) \operatorname{sgn}\left(\sigma^{\prime}, \sigma \cap \sigma^{\prime}\right)=0,
$$

and hence $\ell_{\sigma \sigma^{\prime}}^{\text {up }}(\mathbf{z})+\ell_{\sigma \sigma^{\prime}}^{\text {down }}(\mathbf{z})=0$. If $\sigma=\sigma^{\prime}$, then we have

$$
\begin{aligned}
& \ell_{\sigma \sigma}^{\mathrm{up}}(\mathbf{z})+\ell_{\sigma \sigma}^{\text {down }}(\mathbf{z}) \\
& =\sum_{\eta \in K_{d}: \eta \supset \sigma}\left(z_{\eta \backslash \sigma}^{-1}-1\right)\left(z_{\eta \backslash \sigma}-1\right)+\sum_{\tau \in K_{d-2}: \tau \subset \sigma}\left(z_{\sigma \backslash \tau}-1\right)\left(z_{\sigma \backslash \tau}^{-1}-1\right) \\
& =\sum_{i=1}^{q}\left(z_{i}-1\right)\left(z_{i}^{-1}-1\right) .
\end{aligned}
$$

This completes the proof.

Lemma 2.10. The eigenvalues of $L_{d}^{\mathrm{up}}(\mathbf{z})\left(\operatorname{resp} . L_{d}^{\text {down }}(\mathbf{z})\right)$ on the periodic $q$-cubical lattice $Y$ are $2 q-\sum_{i=1}^{q}\left(z_{i}+z_{i}^{-1}\right)$ with multiplicity $\left(\begin{array}{c}q-1 \\ d\end{array}\right)$ (resp. $\left.\left(\begin{array}{c}q-1 \\ d-1\end{array}\right)\right)$ and 0 with multiplicity $\left(\begin{array}{c}q-1 \\ d-1\end{array}\right)$ (resp. $\left.\left(\begin{array}{c}q-1 \\ d\end{array}\right)\right)$.

Proof. By Corollary 2.9, for any $f \in \operatorname{ker} L_{d}^{\text {down }}(\mathbf{z})$, we have

$$
L_{d}^{\mathrm{up}}(\mathbf{z}) f=\left\{2 q-\sum_{i=1}^{q}\left(z_{i}+z_{i}^{-1}\right)\right\} f .
$$

Also, for any non-zero $f \in \operatorname{ker} L_{d}^{\text {up }}(\mathbf{z})$, by definition, $L_{d}^{\text {up }}(\mathbf{z}) f=0$. Since $\operatorname{dim} \operatorname{ker} L_{d}^{\text {down }}(\mathbf{z})=$ $\operatorname{dim} \operatorname{ker} \delta_{d-1}^{*}(\mathbf{z})=\left(\begin{array}{c}q-1 \\ d\end{array}\right)$ and $\operatorname{dim} \operatorname{ker} L_{d}^{\text {up }}(\mathbf{z})=\operatorname{dim} \operatorname{ker} \delta_{d}(\mathbf{z})=\left(\begin{array}{c}q-1 \\ d-1\end{array}\right)$ by Corollary 2.7, the space $C_{\mathbf{z}}^{d}(Y)$ is spanned by those eigenfunctions.

Corollary 2.11. The eigenvalues of $L_{d}^{\text {up }}$ are given by

$$
\operatorname{Spec}\left(L_{d}^{\mathrm{up}}\right)=\left\{2 q-2 \sum_{i=1}^{q} \cos \frac{2 \pi k_{i}}{n_{i}}: k_{i} \in \mathbb{Z}_{n_{i}}\right\} \cup\{0\} .
$$

The multiplicity of the eigenvalue $2 q-2 \sum_{i=1}^{q} \cos \frac{2 \pi k_{i}}{n_{i}}$ for each $\mathbf{k}=\left(k_{1}, \ldots, k_{q}\right)$ is $\left(\begin{array}{c}q-1 \\ d\end{array}\right)$, and that of 0 is $\left(\begin{array}{c}q-1 \\ d-1\end{array}\right)|\mathbf{n}|$.

Note that the eigenvalue for $\mathbf{k}=(0,0, \ldots, 0)$ with multiplicity $\left(\begin{array}{c}q-1 \\ d\end{array}\right)$ is also 0 , but this eigenvalue is represented within the first set of the right-hand side of (2.2), not the second set.

\section{ZETA FUNCTIONS OF PERIODIC CUBICAL COMPLEXES}

The zeta functions of cubical complexes can be reformulated as those of hypergraphs (see Definition 3.2).

A hypergraph is a pair $H=(V, E)$ of disjoint sets, where $V$ is a non-empty set and the elements of $E$ are non-empty subsets of $V$. An element of $V$ (resp. $E$ ) is called a hypervertex (resp. hyperedge). A hypervertex $v \in V$ is said to be incident to $e \in E$ if $v$ is included in $e$. The $|V| \times|E|$-matrix $M$ indexed by the elements of $V$ and $E$ is defined as $M_{v, e}=1$ if $v$ is incident to $e$; 0 otherwise. This is called the incidence matrix for $H$. The degree, $\operatorname{deg}(v)$, of a hypervertex $v$ is the number of hyperedges that include $v$, and the degree, $\operatorname{deg}(e)$, of a hyperedge $e$ is the number of hypervertices that are included in 
e. A hypergraph $H=(V, E)$ is said to be $(a, b)$-regular if $\operatorname{deg}(v)=a$ for all $v \in V$ and $\operatorname{deg}(e)=b$ for all $e \in E$.

Example 3.1. (1) When $\operatorname{deg}(e)=2$ for every $e \in E$, then a hypergraph is nothing but a graph.

(2) A simplicial complex over a set $V$ can be viewed as a hypergraph by regarding all simplices as $E$.

(3) For a $q$-dimensional cubical complex, let $V$ be the set of $(d-1)$-cubes and $E$ the set of $d$-cubes. Then $H=(V, E)$ forms a hypergraph. In particular, $H=(V, E)$ is a $(2(q-d+1), 2 d)$-regular hypergraph if $V=Y_{d-1}$ and $E=Y_{d}$ for the periodic $q$-cubical lattice $Y$.

A closed path in $H$ is a sequence such that $c=\left(v_{0}, e_{0}, v_{1}, e_{1}, \ldots, v_{n-1}, e_{n-1}\right)$, where $v_{i+1} \in e_{i} \cap e_{i+1}$ and $v_{i} \neq v_{i+1}$ for all $i \in \mathbb{Z}_{n}$. Note that $v_{0} \in e_{n-1} \cap e_{0}$. The length of $c$ is the number of hyperedges in $c$, denoted by $|c|$. We say that a closed path $c$ is a closed geodesic if $e_{i} \neq e_{i+1}$ for all $i \in \mathbb{Z}_{|c|}$. We denote by $c^{m}$ the $m$-multiple of a closed geodesic $c$ formed by $m$ repetetions of $c$. If a closed geodesic $c$ is not expressed as an $m$-multiple of a closed geodesic with $m \geq 2$, then $c$ is said to be prime. Two prime closed geodesics are said to be equivalent if one is obtained from the other through a cyclic permutation. An equivalence class of prime closed geodesics is called a prime cycle. The length of a prime cycle $\mathfrak{p}$ is defined as the length of a representative and is denoted by $|\mathfrak{p}|$. The (Ihara) zeta function of a finite hypergraph is defined as follows.

Definition 3.2. For $u \in \mathbb{C}$ with $|u|$ sufficiently small, the (Ihara) zeta function of a finite hypergraph $H$ is defined by

$$
\zeta_{H}(u)=\prod_{\mathfrak{p} \in P}\left(1-u^{|\mathfrak{p}|}\right)^{-1}
$$

where $P$ is the set of prime cycles of $H$.

The factorization theorem for zeta functions of finite graphs was obtained by H. Bass [1] and a conceptually simpler proof employing oriented linegraph structure was given by Kotani-Sunada [5].

One can associate with a hypergraph $H=(V, E)$ a bipartite graph $B_{H}$ whose vertex partite sets are $V$ and $E$, and the incidence relation gives edges in $B_{H}$, i.e., $V\left(B_{H}\right)=V \sqcup E$ and every edge in $E\left(B_{H}\right)$ connects a vertex in $V$ to one in $E ; v \in V$ and $e \in E$ are joined when $v \in e$. The definition of prime cycles given above fits for the cycle structure of $B_{H}$ when $\operatorname{deg}(v) \geq 2$ for all $v \in V$ as discussed in [6], and hence the theorem for graphs can be extended to the hypergraph setting as follows:

Theorem $3.3([6])$. Let $H=(V, E)$ be a finite, connected hypergraph such that $\operatorname{deg}(v) \geq$ 2 for all $v \in V$ with adjacency matrix $A$ and diagonal degree matrix $D$ in $B_{H}$. Then,

$$
\zeta_{H}(u)=(1-u)^{\chi\left(B_{H}\right)} \operatorname{det}(I-\sqrt{u} A+u Q)^{-1},
$$

where $I$ is the $m \times m$ identity matrix with $m=|V|+|E|, Q=D-I, B_{H}$ is the bipartite graph associated with $H$, and $\chi\left(B_{H}\right)=|V|-|E|$ is the Euler characteristic of $B_{H}$.

In the above theorem, although $\sqrt{u}$ appears in the expression, the zeta function is a rational function of $u$, because the length of the corresponding cycle in $B_{H}$ of a cycle in $H$ is doubled. The colored, oriented linegraph is constructed from $H$ in the proof given in [6], which is based on an idea presented in [5].

The following theorem can also be regarded as a restatement of Hashimoto's theorem on zeta functions of semi-regular graphs because the bipartite graph $B_{H}$ is $(a, b)$-semi-regular 
when $H$ is $(a, b)$-regular. The number of vertices in $B_{H}$ is $|V|+|E|$ and that of edges in $B_{H}$ is $a|V|=b|E|$. We can define the dual hypergraph $H^{*}$ of $H=(V, E)$ by interchanging the roles of partite sets in $B_{H}$, i.e., $H^{*}=\left(E, E_{V}\right)$ with the incidence relation determined by $B_{H}$, where $E_{V}:=\left\{A_{v} \subset E: v \in V\right\}$ with $A_{v}:=\{e \in E: e \ni v\}$ for $v \in V$. Clearly, $H^{*}$ is $(b, a)$-regular if $H$ is $(a, b)$-regular. Also, it is clear that $\zeta_{H}(u)=\zeta_{H^{*}}(u)$ because the cycle structures in $H$ and $H^{*}$ are identical since $B_{H}=B_{H^{*}}$ as graphs.

Theorem $3.4([6])$. Let $H=(V, E)$ be a finite connected $(a, b)$-regular hypergraph with $a, b \geq 2$, and let $M$ be the incidence matrix between $V$ and $E$. Let $\alpha=a-1$ and $\beta=b-1$. Then,

$$
\begin{aligned}
\zeta_{H}(u)^{-1} & =(1-u)^{-\chi\left(B_{H}\right)}(1+\beta u)^{|E|-|V|} \operatorname{det}\left((1+\alpha u)(1+\beta u) I_{V}-u M M^{*}\right) \\
& =(1-u)^{-\chi\left(B_{H}\right)}(1+\alpha u)^{|V|-|E|} \operatorname{det}\left((1+\alpha u)(1+\beta u) I_{E}-u M^{*} M\right),
\end{aligned}
$$

where $\chi\left(B_{H}\right)=|E|-\alpha|V|=|V|-\beta|E|$, and $I_{V}\left(\operatorname{resp} . I_{E}\right)$ is the $|V| \times|V|(\operatorname{resp} .|E| \times|E|)$ identity matrix.

Proof. In Theorem 3.3, the adjacency matrix $A$ and the diagonal matrix $Q$ are expressed by $A=\left(\begin{array}{cc}O & M \\ M^{*} & O\end{array}\right)$ and $Q=\left(\begin{array}{cc}\alpha I_{V} & O \\ O & \beta I_{E}\end{array}\right)$. Using the determinantal identity

$$
\operatorname{det}\left(\begin{array}{ll}
P_{11} & P_{12} \\
P_{21} & P_{22}
\end{array}\right)=\operatorname{det} P_{11} \cdot \operatorname{det}\left(P_{22}-P_{21} P_{11}^{-1} P_{12}\right)
$$

we obtain

$$
\begin{aligned}
& \operatorname{det}(I-\sqrt{u} A+u Q) \\
& =(1+\alpha u)^{|V|} \cdot(1+\alpha u)^{-|E|} \operatorname{det}\left((1+\alpha u)(1+\beta u) I_{E}-u M^{*} M\right) .
\end{aligned}
$$

The second equality is obtained in the same way by changing the roles of 1 and 2 in (3.1).

Theorem 3.4 together with Corollary 2.4 yields the following theorem.

Theorem 3.5. Let $Y$ be a $d$-dimensional cubical lattice with side length $\mathbf{n}=\left(n_{1}, n_{2}, \ldots, n_{q}\right)$ and $Y^{(d)}$ its $d$-skeleton. Let $A_{d-1}^{\text {up }}(\mathbf{z})\left(\operatorname{resp} . A_{d}^{\text {down }}(\mathbf{z})\right)$ on $C_{\mathbf{z}}^{d-1}\left(\operatorname{resp} . C_{\mathbf{z}}^{d}\right)$ be the twisted adjacency operator. Then,

$$
\begin{aligned}
\zeta_{Y^{(d)}}(u)^{-1}= & (1-u)^{\kappa_{d}|\mathbf{n}|}\left(1+\beta_{d} u\right)^{\gamma_{d}|\mathbf{n}|} \\
& \times \prod_{\mathbf{z} \in \hat{V}} \operatorname{det}\left(\left(1+\alpha_{d} u\right)\left(1+\beta_{d} u\right) I_{K_{d-2}}-u A_{d-1}^{\text {up }}(\mathbf{z})\right) \\
= & (1-u)^{\kappa_{d}|\mathbf{n}|}\left(1+\alpha_{d} u\right)^{-\gamma_{d}|\mathbf{n}|} \\
& \prod_{\mathbf{z} \in \hat{V}} \operatorname{det}\left(\left(1+\alpha_{d} u\right)\left(1+\beta_{d} u\right) I_{K_{d-1}}-u A_{d}^{\operatorname{down}}(\mathbf{z})\right),
\end{aligned}
$$

where $\alpha_{d}=2 q-2 d+1, \beta_{d}=2 d-1, \gamma_{d}=\left(\begin{array}{l}q \\ d\end{array}\right)-\left(\begin{array}{c}q \\ d-1\end{array}\right)$ and $\kappa_{d}=(q-d)\left(\begin{array}{c}q \\ d-1\end{array}\right)+(d-1)\left(\begin{array}{l}q \\ d\end{array}\right)$.

Proof. We consider the case in which $V=Y_{d-1}$ and $E=Y_{d}$. Then $H=(V, E)$ is $(2(q-d+1), 2 d)$-regular as in Example 3.1(3) and $-\chi\left(B_{H}\right)=\kappa_{d}|\mathbf{n}|$. Since the incidence matrix $M$ in Theorem 3.4 is set to be $\bigoplus_{\mathbf{z} \in \hat{V}} M_{d-1}(\mathbf{z})$, we have $M_{d-1}(\mathbf{z}) M_{d-1}^{*}(\mathbf{z})=A_{d}^{\text {down }}(\mathbf{z})$, 
and hence

$$
\begin{aligned}
\zeta_{Y^{(d)}}(u)^{-1}= & (1-u)^{\kappa_{d}|\mathbf{n}|}\left(1+\alpha_{d} u\right)^{\left|Y_{d-1}\right|-\left|Y_{d}\right|} \\
& \times \prod_{\mathbf{z} \in \hat{V}} \operatorname{det}\left\{\left(1+\alpha_{d} u\right)\left(1+\beta_{d} u\right) I_{K_{d-1}}-u A_{d}^{\text {down }}(\mathbf{z})\right\},
\end{aligned}
$$

where $\alpha_{d}=2(q-d)+1, \beta_{d}=2 d-1$. The second equality is obtained similarly. This completes the proof.

Remark 3.6. (1) The former expression is useful for $q \geq 2 d-1$, while the latter is useful for $q \leq 2 d-1$ as these two conditions are equivalent to $\gamma_{d} \geq 0$ and $\gamma_{d} \leq 0$, respectively.

(2) The map $d \mapsto q-d+1$ leaves $\alpha_{d}, \beta_{d}$ and $\kappa_{d}$ invariant, and $\gamma_{q-d+1}=-\gamma_{d}$. It follows from this invariance that $\zeta_{Y^{(q-d+1)}}(u)=\zeta_{Y^{(d)}}(u)$.

We obtain Theorem 1.2 as a special case of Theorem 3.5 .

Proof of Theorem 1.2. We consider the case $d=q$, i.e., $V=Y_{q-1}$ and $E=Y_{q}$. Then $H=$ $(V, E)$ is $(2,2 q)$-regular as in Example $3.1(3)$. In this case, $A_{q}^{\text {down }}(\mathbf{z})=M_{q-1}(\mathbf{z}) M_{q-1}^{*}(\mathbf{z})$ is a scalar, and we have $A_{q}^{\text {down }}(\mathbf{z})=\sum_{i=1}^{q} 2\left(1+\cos \theta_{i}\right)$ from Corollary 2.4, where $\theta_{i}=2 \pi k_{i} / n_{i}$. Then, from Theorem 3.5, we have

$$
\begin{aligned}
\zeta_{Y}(u)^{-1}= & (1-u)^{\kappa_{q}|\mathbf{n}|}\left(1+\alpha_{q} u\right)^{\gamma_{q}|\mathbf{n}|} \\
& \times \prod_{k_{1}=1}^{n_{1}} \cdots \prod_{k_{q}=1}^{n_{q}}\left\{\left(1+\alpha_{q} u\right)\left(1+\beta_{q} u\right)-u \sum_{i=1}^{q} 2\left(1+\cos \theta_{i}\right)\right\},
\end{aligned}
$$

where $\alpha_{q}=1, \beta_{q}=2 q-1$ and $\kappa_{q}=\gamma_{q}=q-1$. Therefore, we obtain

$$
\zeta_{Y}(u)^{-1}=\left(1-u^{2}\right)^{(q-1)|\mathbf{n}|} \prod_{k_{1}=1}^{n_{1}} \cdots \prod_{k_{q}=1}^{n_{q}}\left\{1-2 u \sum_{i=1}^{q} \cos \theta_{i}+(2 q-1) u^{2}\right\} .
$$

This completes the proof.

We denote by $e_{k}(\mathbf{t})$ the $k$ th elementary symmetric polynomial of $\mathbf{t}=\left(t_{1}, \ldots, t_{q}\right)$ defined by the expansion formula

$$
\prod_{k=1}^{q}\left(\lambda+t_{k}\right)=\sum_{k=0}^{q} e_{k}(\mathbf{t}) \lambda^{q-k}
$$

Proposition 3.7. Let $w_{i}=2+z_{i}+z_{i}^{-1}$. Then,

$$
\operatorname{det}\left(t-u A_{1}^{\mathrm{up}}(\mathbf{z})\right)=\sum_{k=0}^{q}(2-k) 2^{k-1} e_{k}(\mathbf{w})\left(t-u e_{1}(\mathbf{w})\right)^{q-k} u^{k} .
$$

Proof. From Lemma 2.3, we see that the $(j, j)$-element of the matrix $A_{1}^{\text {up }}(\mathbf{z})-e_{1}(\mathbf{w}) I_{q}$ is given by

$$
\sum_{k \neq j}\left(1+z_{k}^{-1}\right)\left(1+z_{k}\right)-\sum_{k=1}^{q}\left(1+z_{k}^{-1}\right)\left(1+z_{k}^{-1}\right)=-\left(1+z_{j}^{-1}\right)\left(1+z_{j}\right) .
$$

Thus we have

$$
A_{1}^{\mathrm{up}}(\mathbf{z})-e_{1}(\mathbf{w}) I_{q}=D_{12} \mathbf{1}^{-1}\left(J_{q}-2 I_{q}\right) D_{\mathbf{1}+\mathbf{z}}
$$


where $J_{q}$ is the $q \times q$ matrix whose elements are all $1, D_{\mathbf{1 + \mathbf { z }}}$ (resp. $\left.D_{\mathbf{1 + \mathbf { z } ^ { - 1 }}}\right)$ is the diagonal matrix whose $(j, j)$-element is $\left(1+z_{j}\right)$ (resp. $\left.\left(1+z_{j}^{-1}\right)\right)$. Hence, setting $\lambda=t-e_{1}(\mathbf{w})$, we obtain

$$
\begin{aligned}
\operatorname{det}\left(t-A_{1}^{\mathrm{up}}(\mathbf{z})\right) & =\operatorname{det}\left(\lambda-D_{\mathbf{1}+\mathbf{z}^{-1}}\left(J_{q}-2 I_{q}\right) D_{\mathbf{1}+\mathbf{z}}\right) \\
& =\operatorname{det}\left(\lambda-\left(J_{q}-2 I_{q}\right) D_{\mathbf{w}}\right) \\
& =\operatorname{det} D_{\mathbf{w}} \operatorname{det}\left(\left(\lambda D_{\mathbf{w}^{-1}}+2 I_{q}\right)-J_{q}\right) .
\end{aligned}
$$

It is easy to see that for any $\mathbf{a}=\left(a_{1}, \ldots, a_{q}\right)$ the relation

$$
\operatorname{det}\left(D_{\mathbf{a}}-J_{q}\right)=e_{q}(\mathbf{a})-e_{q-1}(\mathbf{a})
$$

holds. Thus we obtain

$$
\operatorname{det}\left(t-A_{1}^{\mathrm{up}}(\mathbf{z})\right)=\left.\left(1-\frac{q}{2}+\frac{\lambda}{2} \frac{\partial}{\partial \lambda}\right) \prod_{i=1}^{q}\left(\lambda+2 w_{i}\right)\right|_{\lambda=t-e_{1}(\mathbf{w})} .
$$

Expanding the right-hand side in $\lambda$ and using (3.2), we reach the desired relation.

Proof of the latter half of Theorem 1.2. A simple calculation shows that

$$
\zeta_{Y^{(2)}}(u)^{-1}=(1-u)^{\kappa_{2}|\mathbf{n}|}(1+3 u)^{\gamma_{2}|\mathbf{n}|} \times \prod_{\mathbf{z} \in \hat{V}} F_{1}^{\mathrm{up}}(u, \mathbf{z}),
$$

where $\kappa_{2}=q(3 q-5) / 2, \gamma_{2}=q(q-3) / 2$ and

$$
F_{1}^{\mathrm{up}}(u, \mathbf{z})=\sum_{k=0}^{q}(2-k) 2^{k-1} e_{k}(\mathbf{w})\left(1-u \sum_{i=1}^{q}\left(z_{i}+z_{i}^{-1}\right)+3(2 q-3) u^{2}\right)^{q-k} u^{k} .
$$

We remark that the right-hand sides of the two expressions in Theorem 1.2 must coincide for $q=2$ by symmetry. Indeed, when $q=2$, we see that $\kappa=1, \gamma=-1$ and $F_{1}^{\mathrm{up}}(u, \mathbf{z})=(1+u)(1+3 u)\left(1-u \sum_{i=1}^{2}\left(z_{i}+z_{i}^{-1}\right)+3 u^{2}\right)$. Thus, in this case, both of these are equal to $\left(1-u^{2}\right)^{|\mathbf{n}|} \prod_{k_{1}=1}^{n_{1}} \prod_{k_{2}=1}^{n_{2}}\left(1-2 u \sum_{i=1}^{2} \cos 2 \pi k_{i} / n_{i}+3 u^{2}\right)$.

\section{Cyclotomic-Like polynomials}

For $\mathbf{d}=\left(d_{1}, \ldots, d_{q}\right) \in \mathbb{N}^{q}$, we define the following polynomial in $x$ :

$$
\Psi_{\mathbf{d}}(x):=\prod_{j_{1} \in J_{d_{1}}} \cdots \prod_{j_{q} \in J_{d_{q}}}\left(x-2 \sum_{i=1}^{q} \cos \frac{2 \pi j_{i}}{d_{i}}\right),
$$

With the notation used in Introduction, this would be written $\Psi_{\mathbf{d}}(x, 1)$, but here, we use the above more concise notation. Since the homogeneous polynomial $\Psi_{\mathbf{d}}(x, y)$ can be recovered from $\Psi_{\mathbf{d}}(x)$, hereafter we focus on $\Psi_{\mathbf{d}}(x)$. We note that $\Psi_{\mathbf{d}}=\Psi_{\mathbf{d}^{\prime}}$ when $\mathbf{d}^{\prime}$ is a permutation of $\mathbf{d}$ and that the degree of $\Psi_{\mathbf{d}}$ is equal to $\prod_{i=1}^{q} \tilde{\varphi}\left(d_{i}\right)$. For $q=1$, there is a known explicit form of $\Psi_{d}(x)$ that can be obtained using cyclotomic polynomials.

Lemma 4.1. Suppose $q=1$. Then $z^{\tilde{\varphi}(d)} \Psi_{d}\left(z+z^{-1}\right)$ is the $d$ th cyclotomic polynomial for $d \geq 3$ and the square of that for $d=1,2$. Moreover, $\Psi_{d}(x)$ is irreducible for any $d \in \mathbb{N}$.

Proof. The proof is trivial for $d=1,2$. Suppose $d \geq 3$. It is easily seen that $z^{\tilde{\varphi}(d)} \Psi_{d}(z+$ $z^{-1}$ ) is a monic polynomial in $z$ of degree $2 \tilde{\varphi}(d)$, since the degree of $\Psi_{d}(x) \in \mathbb{Z}[x]$ is $\tilde{\varphi}(d)$. This polynomial has the following $\varphi(d)=2\left|J_{d}\right|$ distinct roots

$$
\left\{z=\exp (2 \pi \sqrt{-1} j / d) \mid j \in J_{d}\right\} \cup\left\{z=\exp (-2 \pi \sqrt{-1} j / d) \mid j \in J_{d}\right\} .
$$


These properties characterize the cyclotomic polynomial. If $\Psi_{d}(x)$ is reducible, then so is $\Psi_{d}\left(z+z^{-1}\right)$. This contradicts the irreducibility of cyclotomic polynomials.

Example 4.2. $\Phi_{d}(x)$ is the $d$ th cyclotomic polynomial, and $\Psi_{d}(x)$ is that defined in (4.1) for $q=1$. We have the following explicit forms:

$$
\begin{aligned}
\Phi_{1}(x) & =x-1 & \Psi_{1}(x) & =x-2 \\
\Phi_{2}(x) & =x+1 & \Psi_{2}(x) & =x+2 \\
\Phi_{3}(x) & =x^{2}+x+1 & \Psi_{3}(x) & =x+1 \\
\Phi_{4}(x) & =x^{2}+1 & \Psi_{4}(x) & =x \\
\Phi_{5}(x) & =x^{4}+x^{3}+x^{2}+x+1 & \Psi_{5}(x) & =x^{2}+x-1 \\
\Phi_{6}(x) & =x^{2}-x+1 & \Psi_{6}(x) & =x-1 \\
\Phi_{7}(x) & =x^{6}+x^{5}+x^{4}+x^{3}+x^{2}+x+1 & \Psi_{7}(x) & =x^{3}+x^{2}-2 x-1 \\
\Phi_{8}(x) & =x^{4}+1 & \Psi_{8}(x) & =x^{2}-2 \\
\Phi_{9}(x) & =x^{6}+x^{3}+1 & \Psi_{9}(x) & =x^{3}-3 x+1 \\
\Phi_{10}(x) & =x^{4}-x^{3}+x^{2}-x+1 & \Psi_{10}(x) & =x^{2}-x-1
\end{aligned}
$$

Suppose that $d_{1}=1,2,3,4$ or 6 . Then, for $\mathbf{d}^{\prime}=\left(d_{2}, \ldots, d_{q}\right)$, we have $\Psi_{\mathbf{d}}(x)=\Psi_{\mathbf{d}^{\prime}}\left(\Psi_{d_{1}}(x)\right)$ since $J_{d_{1}}=\{1\}$. From this observation, one can compute several $\Psi_{\mathbf{d}}$ s by using the above table.

In Theorem 1.2, the polynomial

$$
F_{\mathbf{n}}(x, y):=\prod_{k_{1}=1}^{n_{1}} \cdots \prod_{k_{q}=1}^{n_{q}}\left(x-2 y \sum_{i=1}^{q} \cos \frac{2 \pi k_{i}}{n_{i}}\right)
$$

appears. This polynomial can be decomposed in terms of $\Psi_{\mathbf{d}}(x, y)$ as $x^{n}-1$ is decomposed into a product of cyclotomic polynomials $\Phi_{d}(x)$.

Proof of Theorem 1.3. Let $\tilde{J}_{d}=\{j \in\{1,2, \ldots, d\}: \operatorname{gcd}(j, d)=1\}$. We note that

$$
\left\{\frac{k}{n} \mid k \in\{1,2, \ldots, n\}\right\}=\bigsqcup_{d \mid n}\left\{\frac{j}{d} \mid j \in \tilde{J}_{d}\right\}
$$

and each set on the right-hand side can be further decomposed as

$$
\left\{\frac{j}{d} \mid j \in \tilde{J}_{d}\right\}= \begin{cases}\left\{\frac{j}{d} \mid j \in J_{d}\right\} \sqcup\left\{\frac{d-j}{d} \mid j \in J_{d}\right\} & \text { for } d \geq 3, \\ \left\{\frac{j}{d} \mid j \in J_{d}\right\} & \text { for } d=1,2 .\end{cases}
$$

Therefore, we have

$$
\begin{aligned}
F_{\mathbf{n}}(x, y) & =\prod_{d_{1} \mid n_{1}} \cdots \prod_{d_{q} \mid n_{q} j_{1} \in J_{d_{1}}} \cdots \prod_{j_{q} \in J_{d_{q}}}\left(x-2 y \sum_{i=1}^{q} \cos \frac{2 \pi j_{i}}{d_{i}}\right)^{\epsilon\left(d_{1}\right) \times \cdots \times \epsilon\left(d_{q}\right)} \\
& =\prod_{d_{1} \mid n_{1}} \cdots \prod_{d_{q} \mid n_{q}} \Psi_{\mathbf{d}}(x, y)^{\epsilon(\mathbf{d})}
\end{aligned}
$$

where $\epsilon(d)=2$ for $d \geq 3$ and $\epsilon(d)=1$ for $d=1,2$. This completes the proof since $\zeta_{Y}(u)^{-1}=\left(1-u^{2}\right)^{(q-1)|\mathbf{n}|} F_{\mathbf{n}}\left(1+(2 q-1) u^{2}, u\right)$. 
Now, in order to factorize $\Psi_{\mathbf{d}}(x)$ further, we consider the orbit structure for Galois actions.

Suppose $d_{i} \geq 3$ for $i=1, \ldots, q$. Let $N=d_{1} \cdots d_{q}$. We identify $J_{d}$ for $d \geq 3$ with the set of representatives of $(\mathbb{Z} / d \mathbb{Z})^{\times}$modulo $x \mapsto-x$. We identify $(\mathbb{Z} / N \mathbb{Z})^{\times}$with $\{m \in \mathbb{N} \mid m<N, \operatorname{gcd}(m, N)=1\}$. The group $(\mathbb{Z} / N \mathbb{Z})^{\times}$acts on $J_{d_{1}} \times \cdots \times J_{d_{q}}$ as component-wise multiplication:

$$
\left(j_{1}, \ldots, j_{q}\right) \mapsto\left(a j_{1}, \ldots, a j_{q}\right)
$$

for $a \in(\mathbb{Z} / N \mathbb{Z})^{\times}$.

Before proving Theorem 1.4, we give the following lemma.

Lemma 4.3. For $\left(d_{1}, d_{2}, \ldots, d_{q}\right)$ with $d_{i} \geq 3$ for all $i=1, \ldots, q$, put $N^{\prime}:=\operatorname{lcm}\left(d_{1}, \ldots, d_{q}\right)$, $G:=\left(\mathbb{Z} / N^{\prime} \mathbb{Z}\right)^{\times}$and

$$
H:=\left\{g \in G \mid \exists\left(\varepsilon_{i}\right) \in\{ \pm 1\}^{q} \text { such that } g \equiv \varepsilon_{i} \quad \bmod d_{i}(i=1, \ldots, q)\right\} .
$$

Then, the quotient group $G / H$ acts on $J_{d_{1}} \times \cdots \times J_{d_{q}}$ freely. In particular, every $G / H$ orbit on $J_{d_{1}} \times \cdots \times J_{d_{q}}$ has $|G / H|$ elements. The cardinality of $H$ is $2^{\beta_{0}(\Gamma(V))}$, where $V=\{1,2, \ldots, q\}$ and $\beta_{0}(\Gamma(V))$ is the 0th Betti number, i.e., the number of connected components of $\Gamma(V)$.

Proof. We express the decomposition into connected components as $\{1, \ldots, q\}=\sqcup_{k=1}^{\beta_{0}} A_{k}$, where $\beta_{0}$ represents $\beta_{0}(\Gamma(V))$. Note that

$$
H=\left\{g \in G \mid \exists\left(\varepsilon_{k}\right) \in\{ \pm 1\}^{\beta_{0}} \text { such that } g \equiv \varepsilon_{k} \quad \bmod d_{i} \text { for } i \in A_{k}\right\} .
$$

In fact, we see that $\varepsilon_{i} \equiv g \equiv \varepsilon_{j} \bmod \operatorname{gcd}\left(d_{i}, d_{j}\right)$. Thus, if $\operatorname{gcd}\left(d_{i}, d_{j}\right) \geq 3$, then $\varepsilon_{i}=\varepsilon_{j}$. This implies the above identity. Put $N_{k}:=\operatorname{lcm}\left(d_{i} \mid i \in A_{k}\right)$ for $k=1, \ldots, \beta_{0}$. Then, we can write

$$
H=\left\{g \in G \mid \exists\left(\varepsilon_{k}\right) \in\{ \pm 1\}^{\beta_{0}} \text { such that } g \equiv \varepsilon_{k} \quad \bmod N_{k}\right\} .
$$

Also, it is seen that $\operatorname{gcd}\left(N_{k}, N_{k^{\prime}}\right) \leq 2$ for $k \neq k^{\prime}$. It follows that the map

$$
H \rightarrow\left(\varepsilon_{k}\right) \in\{ \pm 1\}^{\beta_{0}}
$$

is bijective.

Proof of Theorem 1.4. For simplicity, $\mathbf{d}$ is assumed to be rearranged in such a way that $d_{1} \geq \cdots \geq d_{q^{\prime}} \geq 3>d_{q^{\prime}+1} \geq \cdots \geq d_{q}$, and we write $\mathbf{d}^{\prime}=\left(d_{1}, \ldots, d_{q^{\prime}}\right)$. It is clear that $\operatorname{orb}(\mathbf{d})=\operatorname{orb}\left(\mathbf{d}^{\prime}\right)$ since $J_{1}$ and $J_{2}$ are singletons, and since $\tilde{\varphi}(1)=\tilde{\varphi}(2)=1$ and $\tilde{\varphi}(\operatorname{lcm}(m, 2))=\tilde{\varphi}(m)$ for any $m \in \mathbb{N}$, we have

$$
\frac{\prod_{i=1}^{q} \tilde{\varphi}\left(d_{i}\right)}{\tilde{\varphi}\left(\operatorname{lcm}\left(d_{1}, d_{2}, \ldots, d_{q}\right)\right)}=\frac{\prod_{i=1}^{q^{\prime}} \tilde{\varphi}\left(d_{i}\right)}{\tilde{\varphi}\left(\operatorname{lcm}\left(d_{1}, d_{2}, \ldots, d_{q}^{\prime}\right)\right)} .
$$

Therefore, it suffices to consider the case $d_{i} \geq 3$ for all $i=1,2, \ldots, q$. From Lemma 4.3, it follows that

$$
\operatorname{orb}(\mathbf{d})=\frac{\left|J_{d_{1}} \times \cdots \times J_{d_{q}}\right|}{|G / H|}=\frac{\prod_{i=1}^{q} \tilde{\varphi}\left(d_{i}\right)}{\tilde{\varphi}\left(\operatorname{lcm}\left(d_{1}, d_{2}, \ldots, d_{q}\right)\right)} 2^{\beta_{0}(\Gamma(V))-1} .
$$

This completes the proof.

We next give a criterion for the irreducibility of $\Psi_{\mathbf{d}}(x)$, which is later used to obtain a condition for $\Psi_{\mathbf{d}}(x ; \mathcal{O})$ defined in (1.2) to factor into the powers of a linear function for $q=2$. 
Proposition 4.4. (1) If $\mathcal{O} \subset J_{d_{1}} \times \cdots \times J_{d_{q}}$ is stable under the action of $(\mathbb{Z} / N \mathbb{Z})^{\times}$, then $\Psi_{\mathrm{d}}(x ; \mathcal{O}) \in \mathbb{Z}[x]$.

(2) Let $\mathcal{O} \subset J_{d_{1}} \times \cdots \times J_{d_{q}}$ be a $(\mathbb{Z} / N \mathbb{Z})^{\times}$-orbit. Then the map

$$
c: \mathcal{O} \ni\left(j_{1}, \ldots, j_{q}\right) \mapsto 2 \sum_{i=1}^{q} \cos \frac{2 \pi j_{i}}{d_{i}} \in \mathbb{R}
$$

is injective if and only if $\Psi_{\mathbf{d}}(x ; \mathcal{O}) \in \mathbb{Z}[x]$ is irreducible.

Proof. The Galois group $\operatorname{Gal}\left(\mathbb{Q}\left(\zeta_{N}\right) / \mathbb{Q}\right)$ is identified with $(\mathbb{Z} / N \mathbb{Z})^{\times}$, where $\zeta_{N}=\exp (2 \pi \sqrt{-1} / N)$ is an $N$ th primitive root of unity. The Galois action of an element $a \in(\mathbb{Z} / N \mathbb{Z})^{\times}$to an element $c\left(j_{1}, \ldots, j_{q}\right) \in \mathbb{Q}\left(\zeta_{N}\right)$ is given by the action (4.3). If $\mathcal{O}$ is stable under the action of $(\mathbb{Z} / N \mathbb{Z})^{\times}$, then $\Psi_{\mathbf{d}}(x ; \mathcal{O}) \in \mathbb{Q}[x]$. Since the coefficients of $\Psi_{\mathbf{d}}(x ; \mathcal{O})$ are algebraic integers, we obtain the first assertion (1). If the map $c$ is not injective, then $\Psi_{\mathbf{d}}(x ; \mathcal{O})$ has a multiple root, and hence it cannot be irreducible. Conversely, if the map $c$ is injective on an orbit $\mathcal{O}$, then the action of $(\mathbb{Z} / N \mathbb{Z})^{\times}$on the roots of $\Psi_{\mathbf{d}}(x ; \mathcal{O})$ is transitive, and therefore the polynomial $\Psi_{\mathbf{d}}(x ; \mathcal{O})$ is irreducible.

We now give an example of Proposition 4.4(2).

Corollary 4.5. Suppose $d_{1}, \ldots, d_{q}$ are relatively prime and $d_{i} \geq 3$ for all $i=1,2, \ldots, q$. Then the action of $(\mathbb{Z} / N \mathbb{Z})^{\times}$on $J_{d_{1}} \times \cdots \times J_{d_{q}}$ is transitive, the map $c$ in (4.4) is injective, and $\Psi_{\mathbf{d}}(x) \in \mathbb{Z}[x]$ is irreducible.

Proof. For any $\left(j_{1}, \ldots, j_{q}\right) \in J_{d_{1}} \times \cdots \times J_{d_{q}}$, there exists $g \in \mathbb{Z}$ such that $j_{i} \equiv g$ $\bmod d_{i}$ for all $i=1, \ldots, q$ from the Chinese Remainder Theorem. This shows that $\left(j_{1}, \ldots, j_{q}\right)=(g 1, \ldots, g 1)$ belongs to an orbit of $(1, \ldots, 1)$, and thus the transitivity follows. The injectivity is proved as follows. First, suppose $c\left(j_{1}, \ldots, j_{q}\right)=c\left(j_{1}^{\prime}, \ldots, j_{q}^{\prime}\right)$. Then,

$$
-\cos \left(2 \pi j_{1} / d_{1}\right)+\cos \left(2 \pi j_{1}^{\prime} / d_{1}\right)=\sum_{i=2}^{q}\left(\cos \left(2 \pi j_{i} / d_{i}\right)-\cos \left(2 \pi j_{i}^{\prime} / d_{i}\right)\right)
$$

is an element of $\mathbb{Q}\left(\zeta_{d_{1}}\right) \cap \mathbb{Q}\left(\zeta_{d_{2} \cdots d_{q}}\right)=\mathbb{Q}$ since $d_{1}$ and $d_{2} \cdots d_{q}$ are coprime. There exists a rational number $b$ such that $-\cos \left(2 \pi j_{1} / d_{1}\right)+\cos \left(2 \pi j_{1}^{\prime} / d_{1}\right)=b$. Taking the Galois conjugates, and summing up over $J_{d_{1}}$, we have

$$
\tilde{\varphi}\left(d_{1}\right) b=-\sum_{j_{1} \in J_{d_{1}}} \cos \frac{2 \pi j_{1}}{d_{1}}+\sum_{j_{1}^{\prime} \in J_{d_{1}}} \cos \frac{2 \pi j_{1}^{\prime}}{d_{1}}=0 .
$$

This implies $b=0$ and $j_{1}^{\prime}=j_{1}$. Then, by induction on $q$, we have the injectivity. The irreducibility follows from Proposition 4.4(2).

The polynomial $\Psi_{\mathbf{d}}(x ; \mathcal{O})$ may not be irreducible. The following lemma clarifies the situation in this regard.

Lemma 4.6. Let $\mathcal{O} \subset J_{d_{1}} \times \cdots \times J_{d_{q}}$ be a $(\mathbb{Z} / N \mathbb{Z})^{\times}$-orbit. Then the fibers of the map $c$ in (4.4) have the same cardinality. In particular, there exist an irreducible polynomial $\Psi_{\mathbf{d}}^{\mathrm{irr}}(x ; \mathcal{O}) \in \mathbb{Z}[x]$ and a number $m_{\mathcal{O}} \in \mathbb{N}$ such that $\Psi_{\mathbf{d}}(x ; \mathcal{O})=\Psi_{\mathbf{d}}^{\mathrm{irr}}(x ; \mathcal{O})^{m_{\mathcal{O}}}$.

Proof. Take $\left(j_{10}, \ldots, j_{q 0}\right) \in \mathcal{O}$ so that the cardinality of the fiber of the map (4.4) is maximum. Denote $y_{0}:=c\left(j_{10}, \ldots, j_{q 0}\right) \in \mathbb{R}$. For any $y \in c(\mathcal{O})$, there exists a $g \in$ 
$(\mathbb{Z} / N \mathbb{Z})^{\times}$such that $y=c\left(g j_{10}, \ldots, g j_{q 0}\right)$ since $\mathcal{O}$ is an orbit. We see that the map

$$
c^{-1}\left(y_{0}\right) \ni\left(j_{1}, \ldots, j_{q}\right) \mapsto\left(g j_{1}, \ldots, g j_{q}\right) \in c^{-1}(y)
$$

is a well-defined injective map. By the choice of maximality, this map turns out to be bijective. This proves the first assertion. Letting $m_{\mathcal{O}}$ denote the common cardinality of the fiber and putting

$$
\Psi_{\mathbf{d}}^{\mathrm{irr}}(x ; \mathcal{O})=\prod_{y \in c(\mathcal{O})}(x-y),
$$

we have the second assertion.

Example 4.7. In the case where $q=2$ and $\mathbf{d}=(5,5)$, we have two orbits. One is 'diagonal' and the other is 'off-diagonal':

$$
\mathcal{O}_{1}=\{(1,1),(2,2)\}, \quad \mathcal{O}_{2}=\{(1,2),(2,1)\} .
$$

Note that $c$ in (4.4) maps the orbits as follows:

$$
\mathcal{O}_{1} \mapsto\left\{4 \cos \frac{2 \pi}{5}, 4 \cos \frac{4 \pi}{5}\right\}, \quad \mathcal{O}_{2} \mapsto\{-1,-1\} .
$$

The latter is not injective, while the former is. We see that $\Psi_{\mathbf{d}}\left(x ; \mathcal{O}_{1}\right)=x^{2}+2 x-4$ is irreducible, and $\Psi_{\mathbf{d}}\left(x ; \mathcal{O}_{2}\right)=(x+1)^{2}$ is reducible, that is, $\Psi_{\mathbf{d}}^{\mathrm{irr}}\left(x ; \mathcal{O}_{2}\right)=x+1, m_{\mathcal{O}_{1}}=1$ and $m_{\mathcal{O}_{2}}=2$.

In what follows, we focus on the case $q=2$, in which we know more about the reducibility of $\Psi_{\mathbf{d}}(x ; \mathcal{O})$.

Proposition 4.8. Suppose $q=2$. If the degree of $\Psi_{d_{1}, d_{2}}^{\mathrm{irr}}(x ; \mathcal{O})$ is one, the possibilities for $d_{1}, d_{2}$ and the orbits $\mathcal{O}$ are as follows:

\begin{tabular}{|c|c|c|c|}
\hline$\left(d_{1}, d_{2}\right)$ & $\mathcal{O}$ & $\Psi_{d_{1}, d_{2}}^{\mathrm{irr}}(x ; \mathcal{O})$ & condition \\
\hline \hline$\left(d_{1}, d_{2}\right)$ & $\mathcal{O}_{1,1}$ & $x-\lambda$ & $d_{1}, d_{2}=1,2,3,4,6$ \\
$(m, m)$ & $\mathcal{O}_{1, m / 2-1}$ & $x$ & $4 \mid m$ \\
$(m, 2 m)$ & $\mathcal{O}_{1, m-2}$ & $x$ & $m:$ odd \\
$(5,5)$ & $\mathcal{O}_{1,2}$ & $x+1$ & \\
$(10,10)$ & $\mathcal{O}_{1,3}$ & $x-1$ & \\
\hline
\end{tabular}

In this table, $\lambda=2 \cos \frac{2 \pi}{d_{1}}+2 \cos \frac{2 \pi}{d_{2}} \in \mathbb{Z}$ and $\mathcal{O}_{1, a}$ is the orbit containing $(1, a) \in J_{d_{1}} \times J_{d_{2}}$. More explicitly,

$$
\begin{aligned}
& \mathcal{O}_{1,1}=J_{d_{1}} \times J_{d_{2}}, \quad \mathcal{O}_{1, m / 2-1}=\left\{(j, m / 2-j) \mid j \in J_{m}\right\}, \\
& \mathcal{O}_{1, m-2}=\left\{(j, m-2 j) \mid j \in J_{m}\right\}, \\
& \mathcal{O}_{1,2}, \mathcal{O}_{1,3}=\left\{\left(j_{1}, j_{2}\right) \in J_{d_{1}} \times J_{d_{2}} \mid j_{1} \neq j_{2}\right\} .
\end{aligned}
$$

By Lemma 4.6, if the degree of $\Psi_{d_{1}, d_{2}}^{\mathrm{irr}}(x ; \mathcal{O})$ is one, then the image of the map $c: \mathcal{O} \rightarrow \mathbb{Q}$ is a singleton. We now derive some necessary conditions for this to be the case.

Lemma 4.9. Let $g=\operatorname{gcd}\left(d_{1}, d_{2}\right)$ and define $g_{i}$ to be the product of all factors of $d_{i}$ in common with $g$. Put $m_{i}:=d_{i} / g_{i} \in \mathbb{Z}$. If the degree of $\Psi_{d_{1}, d_{2}}^{\mathrm{irr}}(x ; \mathcal{O})$ is one, or equivalently, if the image of the map $c: \mathcal{O} \rightarrow \mathbb{Q}$ is a singleton, then the following conditions hold:

(i) $g_{i} \leq 2$ or $m_{i} \leq 2$ for $i=1,2$.

(ii) $m_{1}, m_{2} \in\{1,2,3,4,6\}$.

(iii) $g_{1}=g_{2}$ or $\left(g_{1}, g_{2}\right)=(2,4)$ or $(4,2)$. 
Proof. Let $N^{\prime}=\operatorname{lcm}\left(d_{1}, d_{2}\right)$. Suppose there exist $d_{1}, d_{2} \in \mathbb{N}$ and $\left(j_{1}, j_{2}\right) \in J_{d_{1}} \times J_{d_{2}}$ such that

$$
2 \cos \frac{2 \pi j_{1} b}{d_{1}}+2 \cos \frac{2 \pi j_{2} b}{d_{2}}=\lambda \in \mathbb{Q} \quad \text { for } \forall b \in \mathbb{Z} / N^{\prime} \mathbb{Z}
$$

We claim that every $b \in \mathbb{Z}$ with $b \equiv 1 \bmod d_{1}$ and $\operatorname{gcd}\left(b, d_{2}\right)=1$ satisfies $b \equiv \pm 1$ $\bmod d_{2}$. Indeed, since $\cos \frac{2 \pi j_{1} b}{d_{1}}=\cos \frac{2 \pi j_{1}}{d_{1}}$, we have $\cos \frac{2 \pi j_{2} b}{d_{2}}=\cos \frac{2 \pi j_{2}}{d_{2}}$, which implies that $b \equiv \pm 1 \bmod d_{2}$.

Now we use the claim above for three cases regarding the value of $b$. Note that $d_{i}=m_{i} g_{i}$, and if $\tilde{g}=\operatorname{lcm}\left(g_{1}, g_{2}\right)$, then $\tilde{g}, m_{1}$ and $m_{2}$ are mutually prime.

First, consider $b \in \mathbb{Z}$ with $b \equiv 1 \bmod m_{1} \tilde{g}$ and $b \equiv-1 \bmod m_{2}$. By the claim, we have $b \equiv \pm 1 \bmod d_{2}=g_{2} m_{2}$. If $b \equiv 1$, then we have $m_{2} \leq 2$, while if $b \equiv-1$, then we have $g_{2} \leq 2$. We conclude that either $m_{2} \leq 2$ or $g_{2} \leq 2$.

Second, consider $b \in \mathbb{Z}$ with $b \equiv 1 \bmod m_{1} \tilde{g}$ and $\operatorname{gcd}\left(b, m_{2}\right)=1$. By the claim, we have $b \equiv \pm 1 \bmod m_{2}$. This shows that $\varphi\left(m_{2}\right) \leq 2$, and we conclude that $m_{2}=1,2,3,4$ or 6 .

Third, consider $b \in \mathbb{Z}$ with $b \equiv 1 \bmod m_{1} m_{2}$ and $b \equiv 1+g_{1} \bmod g_{2}$. By the claim, we have $b \equiv \pm 1 \bmod g_{2}$. If $b \equiv 1$, then $g_{2} \mid g_{1}$, while if $b \equiv-1$, then $g_{2} \mid\left(g_{1}+2\right)$. Since the conditions on $d_{1}$ and $d_{2}$ are symmetric, we also have $g_{1} \mid g_{2}$ or $g_{1} \mid\left(g_{2}+2\right)$. We conclude that either $g_{1}=g_{2}$ or $\left\{g_{1}, g_{2}\right\}=\{2,4\}$.

Now we use Lemma 4.9 (i), (ii) and (iii) to prove Proposition 4.8 .

Proof of Proposition 4.8 . First, we note that $\Psi_{d_{1}, d_{2}}(x)=\Psi_{d_{2}}(x-\alpha)$ with $\alpha=2 \cos \frac{2 \pi}{d_{1}} \in \mathbb{Z}$ for $d_{1} \in\{1,2,3,4,6\}$ from the remark in Example 4.2. Since $\Psi_{d}(x)$ is irreducible for any $d \in \mathbb{N}$ from Lemma 4.1, so is $\Psi_{\mathbf{d}}(x)$ in this case. The degree of $\Psi^{\mathrm{irr}}(x ; \mathcal{O})$ is one if and only if that of $\Psi_{d_{2}}(x)$ is one, which is true if and only if $d_{2} \in\{1,2,3,4,6\}$. This gives the first line in the table. Indeed, in all of the cases above, we have only one orbit $\mathcal{O}_{1,1}$ on $J_{d_{1}} \times J_{d_{2}}$.

In what follows, we can assume that $d_{1}, d_{2} \notin\{1,2,3,4,6\}$, and hence $\tilde{\varphi}\left(d_{1}\right), \tilde{\varphi}\left(d_{2}\right) \geq 2$.

In the case $g=1$ with $g_{1}=g_{2}=g$ in (iii), we have $m_{i}=d_{i} \in\{1,2,3,4,6\}$ by (ii). In the case $g_{i}=2$ in (iii) for $i=1$ or $2, m_{i}$ should be odd, and hence choices are only $m_{i}=1,3$ by (ii). This implies $d_{i}=2$ or 6 . Both of these cases have already been excluded.

If $g \geq 3$ with $g_{1}=g_{2}=g$, then $m_{i} \leq 2$. The only choices in this case are $\left(m_{1}, m_{2}\right)=$ $(1,1),(1,2),(2,1)$. Furthermore, if $m_{i}=2$, then $g$ must be odd. It follows that $\left(d_{1}, d_{2}\right)=$ $(g, 2 g)$ with odd $g \geq 5$ or $\left(d_{1}, d_{2}\right)=(g, g)$ for arbitrary $g \geq 5$ (with $g=6$ excluded).

To this point, we have not used the orbit structure. Now we consider the choice of $\left(j_{1}, j_{2}\right) \in \mathcal{O}$. Taking the sum of all the terms under the Galois action of $\left(\mathbb{Z} / d_{2} \mathbb{Z}\right)^{\times}$, and using the same argument as in the proof of Corollary 4.5, we have

$$
\tilde{\varphi}\left(d_{2}\right) \lambda=2 \sum_{j_{1} \in J_{d_{1}}} \cos \frac{2 \pi j_{1}}{d_{1}}+2 \sum_{j_{2} \in J_{d_{2}}} \cos \frac{2 \pi j_{2}}{d_{2}}=\mu\left(d_{1}\right)+\mu\left(d_{2}\right)
$$

for $d_{1}, d_{2} \geq 3$, where $\mu$ is the Möbius function. Since $\lambda$ is an algebraic integer, we see that $\mathbb{Z} \ni \lambda=\frac{\mu\left(d_{1}\right)+\mu\left(d_{2}\right)}{\tilde{\varphi}\left(d_{2}\right)}$. This shows either $\lambda=0$ or $\tilde{\varphi}\left(d_{2}\right) \leq 2$.

If $\lambda \neq 0$, then $\tilde{\varphi}\left(d_{2}\right)=2$, i.e., $d_{2} \in\{5,8,10,12\}$. In this case, we have $\lambda=\mu\left(d_{1}\right)=$ $\mu\left(d_{2}\right)= \pm 1$, and thus $d_{2}$ is not a multiple of 4 and $\left(d_{1}, d_{2}\right) \neq(g, 2 g)$. From this we obtain $d_{1}=d_{2} \in\{5,10\}$ and the last two lines in the table.

Now we consider the case $\lambda=0$. The condition $2 \cos \frac{2 \pi j_{1}}{d_{1}}+2 \cos \frac{2 \pi j_{2}}{d_{2}}=0$ implies $j_{1} / d_{1}+j_{2} / d_{2}=1 / 2$ since $d_{1}, d_{2} \geq 3$. If $d_{1}=d_{2}$, then $j_{1}+j_{2}=d_{1} / 2$. It follows that $d_{1}$ is even, and hence that $j_{1}$ and $j_{2}$ are odd. This implies that $d_{1} / 2$ is even, and therefore 
$4 \mid d_{1}$. For $j_{1}=1$, we have $j_{2}=d_{1} / 2-1 \in J_{d_{2}}$. This gives the second line in the table. If $2 d_{1}=d_{2}$, then $2 j_{1}+j_{2}=d_{1}$. For $j_{1}=1$, we have $j_{2}=d_{1}-2 \in J_{d_{2}}$. This gives the third line in the table.

Now we give three examples for $q=2$ : (I) $\left(d_{1}, d_{2}\right)=(m, 2 m) ;(\mathrm{II})\left(d_{1}, d_{2}\right)=(m, m)$; (III) $\tilde{\varphi}\left(d_{2}\right)=2$. From these examples, we obtain three observations with the aid of numerical computations.

Before studying each case, we give an elementary remark on the representatives for an orbit decomposition.

Lemma 4.10. Suppose that $G$ acts on $X$ simply transitively. Fix an arbitrary $x_{0} \in X$. Then, for any $Y$ (with $G$ action), the set $\left\{x_{0}\right\} \times Y$ consists of complete representatives of the action of $G$ on $X \times Y$.

Proof. For any $(x, y) \in X \times Y$, there exists a $g \in G$ such that $g x=x_{0}$. Then $g(x, y)=$ $\left(x_{0}, g y\right)$. If $\left(x_{0}, y\right)$ and $\left(x_{0}, y^{\prime}\right)$ belong to the same $G$-orbit, then $y=y^{\prime}$, because $g\left(x_{0}, y\right)=$ $\left(x_{0}, y^{\prime}\right)$ implies $g=e$, the identity element of the group $G$.

(I) For $q=2,\left(d_{1}, d_{2}\right)=(m, 2 m)$.

We start with the orbit decomposition.

Lemma 4.11. The number of orbits is $\operatorname{orb}((m, 2 m))=\tilde{\varphi}(m)$, and each orbit has $\tilde{\varphi}(2 m)$ elements. The orbit decomposition is given by

$$
J_{m} \times J_{2 m}= \begin{cases}\sqcup_{a \in J_{m}} \mathcal{O}_{1, a} & \text { for even } m, \\ \sqcup_{a \in J_{2 m}} \mathcal{O}_{1, a} & \text { for odd } m .\end{cases}
$$

Proof. The first part follows from Example 1.5 (i) and the second part follows from Lemma 4.10. If $m \geq 3$ is odd, then $\tilde{\varphi}(2 m)=\tilde{\varphi}(m)$ and the natural map induces the group isomorphism $(\mathbb{Z} / 2 m \mathbb{Z})^{\times} /\{ \pm 1\} \stackrel{\sim}{\rightarrow}(\mathbb{Z} / m \mathbb{Z})^{\times} /\{ \pm 1\}$, so the action of $(\mathbb{Z} / 2 m \mathbb{Z})^{\times} /\{ \pm 1\}$ on $J_{m}$ is simply transitive. If $m$ is even, it is easlily seen that $(1, i) \in \mathcal{O}$ if and only if $(1, m-i) \in \mathcal{O}$ for $i \in J_{2 m}$, from which we have the decomposition.

Observation 4.12. (i) $\Psi_{m, 2 m}(x ; \mathcal{O})$ seems to be irreducible except for odd $m$ with $\mathcal{O}=$ $\mathcal{O}_{1, m-2}$ appearing in Proposition 4.8 .

(ii) If $\Psi_{m, 2 m}(x ; \mathcal{O})=\Psi_{m, 2 m}\left(x ; \mathcal{O}^{\prime}\right)$, then $\mathcal{O}=\mathcal{O}^{\prime}$

(II) For $q=2,\left(d_{1}, d_{2}\right)=(m, m)$.

We define the involution $\iota$ on $J_{m} \times J_{m}$ by $\iota(x, y)=(y, x)$. It is obvious from this definition that $\Psi_{m, m}(x ; \iota(\mathcal{O}))=\Psi_{m, m}(x ; \mathcal{O})$.

Lemma 4.13. (i) The number of orbits is $\operatorname{orb}((m, m))=\tilde{\varphi}(m)$ and each orbit has $\tilde{\varphi}(m)$ elements. The orbit decomposition is given by

$$
J_{m} \times J_{m}=\bigsqcup_{a \in J_{m}} \mathcal{O}_{1, a}
$$

(ii) The diagonal orbit $\mathcal{O}_{1,1}=\left\{(i, i): i \in J_{m}\right\}$ is invariant under $\iota$. If $\tilde{\varphi}(m)$ is odd, then there is no non-diagonal $\iota$-invariant orbit.

Proof. (i) The first part follows from Example 1.5. The second part follows from Lemma 4.10 by setting $x_{0}=1$ and $Y=J_{m}$.

(ii) Any non-diagonal $\iota$-invariant orbit has no fixed point under the action of $\iota$. In particular, the cardinality of such an orbit is even. The second assertions follow. 
If $\tilde{\varphi}(m)$ is even, then the situation is different. Note that if $4 \mid m$ with $m>4$, then $\tilde{\varphi}(m)$ is even.

Lemma 4.14. (i) Suppose $4 \mid m$. The orbit $\mathcal{O}_{1, m / 2-1}$ is $\iota$-invariant and $\Psi_{m, m}\left(x ; \mathcal{O}_{1, m / 2-1}\right)=$ $x^{\tilde{\varphi}(m)}$.

(ii) Suppose $\tilde{\varphi}(m)$ is even. For a non-diagonal $\iota$-invariant orbit $\mathcal{O}$ with $\mathcal{O} \neq \mathcal{O}_{1, m / 2-1}$, there exists $\Psi_{m, m}^{(\text {half })}(x ; \mathcal{O}) \in \mathbb{Z}[x]$ of degree $\tilde{\varphi}(m) / 2$ such that $\Psi_{m, m}(x ; \mathcal{O})=\Psi_{m, m}^{(\text {half }}(x ; \mathcal{O})^{2}$.

Proof. (i) This case is considered in Proposition 4.8,

(ii) This can be shown in the same way as the proof of Lemma 4.13 (ii).

We denote by $A$ the number of $\iota$-invariant orbits. As we have seen, $A=1$ if $\tilde{\varphi}(m)$ is odd. We have the following formula for $A=A(m)$ if $\tilde{\varphi}(m)$ is even:

Lemma 4.15. Suppose $\tilde{\varphi}(m)$ is even. Put

$$
f_{1}=f_{1}(m):= \begin{cases}0 & \text { if } 4 \nmid m, \\ 1 & \text { if } 4 \mid m \text { and } 8 \nmid m, \\ 2 & \text { if } 8 \mid m .\end{cases}
$$

Let $f_{2}$ be the number of odd prime factors of $m$. Let $f_{3}=f_{3}(m)=1$ if $4 \nmid m, f_{2}(m) \geq 1$, and $p \equiv 1 \bmod 4$ for every odd prime factor $p$ of $m$; otherwise, we put $f_{3}(m)=0$. Then we have

$$
A=2^{f_{1}+f_{2}+f_{3}-1}
$$

Proof. We consider the orbits that are invariant under the involution $\iota$. We will solve the equation $x^{2} \equiv \pm 1 \bmod m$. By the Chinese Remainder Theorem, this equation can be reduced to the equation for each prime factor. If $m=2^{e}$, then the number of solutions to the equation $x^{2} \equiv 1 \bmod m$ is given by $2^{f_{1}(m)}$. If $m=p^{2}$, then we have two solutions to $x^{2} \equiv 1 \bmod m$. This contributes to $f_{2}$. We have a solution to the equation $x^{2} \equiv-1$ mod $m$ only in the case $f_{3}(m)=1$. This solution makes double the number of solution for the equation $x^{2} \equiv \pm 1 \bmod m$ to the number of solutions to the equation $x^{2} \equiv 1$ mod $m$. Finally, we parameterize the orbits as $\mathcal{O}_{1, x}$ with $x \in J_{m}$, so that we will divide by 2 . The formula for $A(m)$ given above follows.

We now proceed from orbits to polynomials.

Observation 4.16. (i) For an orbit $\mathcal{O} \neq \iota(\mathcal{O}), \Psi_{m, m}(x ; \mathcal{O})$ seem to be irreducible. In particular, $\Psi_{m, m}(x ; \mathcal{O})$ seem to be irreducible if $\tilde{\varphi}(m)$ is odd.

(ii) $\Psi_{m, m}^{\text {(half) }}(x ; \mathcal{O})$ seems to be irreducible for every $\iota$-invariant orbit $\mathcal{O} \neq \mathcal{O}_{1, m / 2-1}$. This happens only if $\tilde{\varphi}(m)$ is even.

(iii) If $\Psi_{m, m}(x ; \mathcal{O})=\Psi_{m, m}\left(x ; \mathcal{O}^{\prime}\right)$, then $\mathcal{O}^{\prime}=\mathcal{O}$ or $\mathcal{O}^{\prime}=\iota(\mathcal{O})$.

(III) For $q=2, \tilde{\varphi}\left(d_{2}\right)=2$.

In this case, $\tilde{\varphi}\left(d_{2}\right)=2$ implies $d_{2}=5,8,10$ or 12 , for which we set $a=2,3,3$ and 5 , respectively. Then $J_{d_{2}}=\{1, a\}$.

Lemma 4.17. Suppose $d_{2}=5,8,10$ or 12 . If $5 \mid d_{1}$ for $d_{2}=10$ or $d_{2} \mid d_{1}$ for $d_{2} \neq 10$, then we have

$$
J_{d_{1}} \times J_{d_{2}}=\mathcal{O}_{1,1} \sqcup \mathcal{O}_{1, a} .
$$

Otherwise, $J_{d_{1}} \times J_{d_{2}}$ forms a single orbit. 
Proof. In these cases, we know that $\operatorname{orb}\left(d_{1}, d_{2}\right)=\tilde{\varphi}\left(d_{2}\right)=2$ from Example 1.5(i). Therefore, the orbit decomposition must be the one given above.

Observation 4.18. If $\tilde{\varphi}\left(d_{2}\right)=2$, then $\Psi_{d_{1}, d_{2}}(x ; \mathcal{O})$ seems to be irreducible except in the cases considered in Proposition 4.8.

\section{Acknowledgment}

This work was partially supported by JST CREST Mathematics 15656429. H.O. is supported in part by JSPS Grants-in-Aid $15 \mathrm{H} 03613$ and 17K18726, and T.S. is supported in part by JSPS Grants-in-Aid 17K18740 and 18H01124.

\section{REFERENCES}

[1] H. Bass. The Ihara-Selberg zeta function of a tree lattice. Internat. J. Math. 3 (1992), 717-797.

[2] D. Boyd. Mahler's measure and special values of $L$-functions. Exp. Math. 7 (1998), 37-82.

[3] K. Hashimoto. Zeta functions of finite graphs and representations of p-adic groups. in Adv. Stud. Pure Math. Vol.15, pp.211-280, Academic Press, New York (1989).

[4] T. Kaczynski, K. Mischaikow, M. Mrozek. Computational homology. Appl. Math. Sci. 157. Springer. 2004.

[5] M. Kotani and T. Sunada. Zeta functions of finite graphs. J. Math. Sci. Univ. Tokyo 7 (2000), 7-25.

[6] C. K. Storm. The zeta function of a hypergraph. Electron. J. Combin. 13 (2006), \#R84.

[7] W. Watkins and J. Zeitlin. The minimal polynomial of $\cos (2 \pi / n)$, Amer. Math. Monthly 100, 5 (1993) 471-474.

Kyoto University Institute for Advanced Study, WPI Institute for the Advanced Study of Human Biology, Kyoto University, Yoshida Ushinomiya-cho, Sakyo-ku, Kyoto 606-8501, Japan; Center for Advanced Intelligence Project, RIKen, 1-4-1 Nihonbashi, CHUO-KU, TOKYO, 103-0027, JAPAN

E-mail address: hiraoka.yasuaki.6z@kyoto-u.ac.jp

Institute of Mathematics for Industry, Kyushu University, 744 Motooka, Nishi-Ku, FUKUOKA 819-0395, JAPAN

E-mail address: ochiai@imi.kyushu-u.ac.jp

Institute of Mathematics for Industry, Kyushu University, 744 Motooka, Nishi-Ku, Fukuoka 819-0395, Japan; Center for Advanced Intelligence Project, RIKen, 1-4-1 NiHONBAShi, Chuo-Ku, TOKYo, 103-0027, JAPAN

E-mail address: shirai@imi.kyushu-u.ac.jp 